\title{
Compound-specific amino acid isotopic proxies for distinguishing between terrestrial and aquatic resource consumption
}

\author{
Emily C. Webb ${ }^{1}$ • Noah V. Honch ${ }^{1}$ Philip J. H. Dunn ${ }^{1}$ - Anna Linderholm ${ }^{2,3}$ • \\ Gunilla Eriksson $^{2} \cdot$ Kerstin Lidén $^{2} \cdot$ Richard P. Evershed $^{1}$
}

Received: 3 March 2015 / Accepted: 15 December 2015 / Published online: 21 April 2016

(C) The Author(s) 2016. This article is published with open access at Springerlink.com

\begin{abstract}
Compound-specific amino acid carbon-isotope compositions have shown particular promise for elucidating dietary behaviors in complex environmental contexts, and may also be able to mitigate the effect of many of the limitations inherent to palaeodietary reconstructions. Here, we investigate the efficacy of compound-specific amino acid isotopic proxies in characterizing the consumption of different dietary protein sources using amino acid carbon-isotope compositions for humans and fauna from Rössberga (Early to Middle Neolithic), Köpingsvik (Mesolithic and Middle Neolithic), and Visby (Medieval Period), Sweden. We also assess the explanatory capabilities of an isotopic mixing model when used with essential amino acid carbon-isotope compositions of humans and local fauna. All three isotopic proxies distinguished among humans from the three sites consistently and informatively, and were able to enhance the broad interpretations made using bulk isotopic compositions. The mixing model palaeodietary reconstruction revealed considerable diversity in relative protein source contributions among individuals at both Köpingsvik and Visby. Comparing the mixing model for bulk carbon- and nitrogen-isotope compositions to the model for essential amino acid isotopic compositions further demonstrated the likelihood of underestimation and overestimation of marine protein consumption for both aquatic-
\end{abstract}

Richard P. Evershed

R.P.Evershed@bristol.ac.uk

1 Organic Geochemistry Unit, School of Chemistry, University of Bristol, Cantock's Close, Bristol, UK BS8 1TS

2 Archaeological Research Laboratory, Stockholm University, SE-106 91 Stockholm, Sweden

3 Research Laboratory for Archaeology, University of Oxford, Dyson Perrins Building, South Parks Road, Oxford, UK OX1 3QY dominant and mixed marine-terrestrial diets when using bulk isotopic compositions.

Keywords Amino acids · Carbon isotopes · Neolithic · Palaeodiet $\cdot$ Mixing models $\cdot$ Sweden

\section{Introduction}

Carbon- and nitrogen-isotope analyses of archaeological human and faunal remains have made important contributions to palaeodietary and palaeoecological reconstruction for decades, but, in recent years, there has been a growing interest in using compound-specific amino acid isotopic analysis to extend the limits of palaeodietary interpretation (Choy et al. 2010; Corr et al. 2005, 2008; Fogel and Tuross 2003; McCullagh et al. 2006; Naito et al. 2010; Smith et al. 2009; Styring et al. 2010, 2015). Amino acid carbon-isotope compositions have shown promise for elucidating dietary behaviors in complex socioeconomic and environmental contexts and may also be able to mitigate the effect of many of the limitations inherent to palaeodietary reconstructions that use bulk collagen isotopic compositions (inter alia Ambrose and Norr 1993; DeNiro and Epstein 1978, 1981; Froehle et al. 2010; Hedges 2004; Koch and Phillips 2002). Although numerous factors impose important limitations on palaeodietary reconstruction from bulk protein isotopic compositions, of particular importance here are the potential for variable tissue-diet isotopic offsets, the effect of the nonprotein portion of diet on body protein isotopic composition, and the influence of both nutritional quality (i.e., ratio of carbohydrates, lipids, and proteins in diet) and protein source (e.g., terrestrial vs. aquatic; Hedges 2004; Howland et al. 2003; Parkington 1991). 
When diet is protein-sufficient, the $\delta^{13} \mathrm{C}$ values of proteinaceous consumer tissues largely reflect the carbon-isotope composition of dietary protein (Ambrose 1993; Kellner and Schoeninger 2007). At higher levels of protein consumption, dietary amino acids are typically routed more or less directly from diet to tissue with minimal modification. When protein consumption is low, carbon drawn from dietary carbohydrates and lipids can constitute $49-58 \%$ of the carbon in collagen (Howland et al. 2003). Crucially, the ability to detect small amounts of marine protein consumption is highly problematic. Preliminary models suggest that a contribution of 20\% marine protein to total dietary protein may cause very small (approximately +0.3 to $+1.8 \%$ o changes in bulk collagen $\delta^{13} \mathrm{C}$ values (Hedges 2004). Thus, if a small amount of marine protein is consumed, with the remainder of total dietary protein derived from a terrestrial source, the carbon-isotope compositions of collagen $\left(\delta^{13} \mathrm{C}_{\mathrm{col}}\right)$ may be quite low, obscuring the marine carbon contribution. In contrast, protein-adequate diets that include large contributions of carbon from marine sources may overestimate marine protein consumption due to preferential routing of marine protein amino acids to body tissues (Corr et al. 2005; Hedges 2004). Nitrogen-isotope compositions can, in some instances, be used to disentangle aquatic and terrestrial resource consumption. Freshwater and marine food webs are considerably more complex than terrestrial food webs and have many trophic levels, which result in higher $\delta^{15} \mathrm{~N}$ values due to cumulative ${ }^{15} \mathrm{~N}$-enrichment from producers to increasingly higher-level consumers. Nondietary factors, however, can have significant impact on the $\delta^{15} \mathrm{~N}$ values of humans, other consumers, and primary producers. Biological factors such as illness, traumatic injury, starvation, rapid growth, physiological stress, and pregnancy can all cause changes in nitrogen metabolism and thus in human and faunal tissue nitrogen-isotope compositions (Fuller et al. 2004a, b; 2005; Heaton et al. 1986; Hedges and Reynard 2007; Hobson et al. 1993; Mekota et al. 2006; Olsen et al. 2014; Petzke et al. 2006; Schwarcz et al. 1999; White and Armelagos 1997; Williams et al. 2011). Aridity and the application of animal dung as manure can also increase the $\delta^{15} \mathrm{~N}$ values of plants and thus increase consumer tissue $\delta^{15} \mathrm{~N}$ values (Bogaard et al. 2007; Fraser et al. 2011; Gröcke et al. 1997).

Isotopic mixing models are commonly used in ecological research to quantify the contributions to diet of different resources for an individual or group, with the objective of elucidating the relationships between producers and consumers in an ecosystem. In order to integrate large, complex datasets and refine insights into human palaeodiet and ancient ecosystems, many archaeologists have also begun making use of the growing body of archaeological and modern isotopic data to apply isotopic mixing models adapted from ecological studies. There are some important limitations associated with mixing models in general (reviewed in Phillips 2012), and their particular application to archaeological contexts. There is uncertainty regarding the expected isotopic offset between carbon in consumed food and in different tissues in the body (i.e., tissue-diet isotopic discrimination). Part of this uncertainty is related to the extent of dietary routing of nonessential amino acids, which may change depending on the kind of dietary protein consumed (e.g., terrestrial vs. marine; Corr et al. 2005), as well as the differences in fractional contributions of different macronutrients (e.g., dietary amino acids, fatty acids, and carbohydrates; Jim et al. 2006) to the protein component of different tissues. The tissue protein carbonisotope composition is a weighted average of the isotopic compositions of both its essential and nonessential amino acids (herein $\delta^{13} \mathrm{C}_{\mathrm{E}-\mathrm{AA}}$ and $\delta^{13} \mathrm{C}_{\mathrm{NE}-\mathrm{AA}}$, respectively). Essential amino acids cannot be biosynthesized by the body and therefore must be ingested in sufficient quantities. In contrast, nonessential amino acids can be assimilated directly from dietary protein, or may be synthesized de novo using components drawn from the body's biochemical pools; the latter process will result in isotopic discrimination or fractionation (Ambrose and Norr 1993). The tissue $\delta^{13} \mathrm{C}_{\mathrm{E}-\mathrm{AA}}$ values are thus expected to closely approximate dietary $\delta^{13} \mathrm{C}_{\mathrm{E}-\mathrm{AA}}$ values due to direct routing, i.e., $\Delta{ }^{13} \mathrm{C}_{\text {tissue }} \mathrm{E}-\mathrm{AA}$ - diet $\mathrm{E}$ ${ }_{\mathrm{AA}} \approx 0 \%$ o (Hare et al. 1991; Howland et al. 2003; Jim et al. 2006). Because essential amino acids are not significantly modified by the body before incorporation into tissues, the tissue $\delta^{13} \mathrm{C}_{\mathrm{E}-\mathrm{AA}}$ values also reflect the isotopic composition of carbon from plants at the base of the food web. Nonessential amino acid $\delta^{13} \mathrm{C}$ values, however, may show evidence of both direct routing and biosynthesis, depending on the quality, digestibility, and amino acid composition of consumed food. Further, although the model can incorporate local modern or archaeological faunal isotopic compositions, there is also, as with all palaeodietary research, a risk of incomplete representation of dietary resources in the archaeological record. Finally, human food choice is extremely variable and often mediated by nonecological or nonbiological factors. Even if a resource is readily available, it may only be consumed by one group within the larger population (e.g., based on gender, status, or socioeconomic role), or perhaps not at all (e.g., due to taste preferences or taboos). Nonetheless, the potential for fine-grained insights and more intuitively understandable dietary reconstructions has led to a number of recent studies investigating archaeological applications of mixing models.

Here, the objective is to investigate the efficacy of compound-specific amino acid carbon isotopic proxies in characterizing the consumption of different dietary protein sources. We present new amino acid carbon-isotope compositions for humans and fauna from three archaeological sites in Sweden dating from the Middle Neolithic to the Medieval period and compare them with previously published bulk isotopic compositions. Archaeological and zooarchaeological data strongly indicate that the inhabitants of each site 
consumed a distinct diet: at Rössberga, human diet was dominated by $\mathrm{C}_{3}$ plant-derived protein; at Köpingsvik, by marine protein; and at Visby, a mixed diet including both $\mathrm{C}_{3}$ plant and marine protein was consumed. This diverse range of diets provides an ideal opportunity to test the ability of amino acid carbon-isotope compositions to describe and differentiate among diets dominated by one class of resources (Rössberga and Köpingsvik) and a heterogeneous mix of dietary protein sources (Visby). We also investigate the explanatory capabilities of IsoSource, a commonly used mixing model, when it is used with essential amino acid carbon-isotope compositions from humans and local fauna. We use the mixing model to broadly quantify the relative contributions to diet of different protein sources for humans from Köpingsvik and Visby, as groups and for each individual, and we compare the outcome of the compound-specific amino acid model to a model generated using the bulk carbon and nitrogen isotopic compositions.

\section{Materials and methods}

\section{Archaeological sampling}

Human and faunal remains were sampled from the sites of Rössberga (Middle to Late Neolithic), Köpingsvik (Middle Neolithic, 3300-2300 BCE), and Visby (Medieval Period) in Sweden (Fig. 1, Table 1). The site of Rössberga includes an Early to Middle Neolithic passage grave containing at least 17 burial compartments and the disarticulated remains of at least 128 individuals, including males, females, and juveniles (Ahlström 2001). It is likely that the humans interred at Rössberga represent a group of pastoralists, most likely engaged in a combination of animal rearing (e.g., cattle and sheep/goats) and hunting (Lidén 1995). Bone and dentin samples from ten humans, as well as several cattle, dog, mountain hare, pig, and red fox specimens, were selected for compoundspecific isotopic analysis. Previous bulk stable isotope analysis suggests reliance on $\mathrm{C}_{3}$ plant-derived terrestrial protein for all individuals $\left({ }^{13} \mathrm{C}-21 \text { to }-20 \% \text {; } \delta^{15} \mathrm{~N}+9 \text { to }+12 \% \circ\right)^{1}$.

Köpingsvik is located on the west coast of Öland Island in the Baltic Sea. The archaeological site has been continuously occupied since the Late Mesolithic. Here, nine individuals attributed to the Middle Neolithic Pitted Ware Culture and one dated to the Mesolithic (Eriksson et al. 2008) were selected for compound-specific isotopic analysis. Numerous faunal remains representing a variety of dietary ecologies were also analyzed, including terrestrial ruminants (cattle, moose,

\footnotetext{
${ }^{1}$ Bulk carbon- and nitrogen-isotopic composition ranges reported in this section reflect all individuals sampled for isotopic analysis, i.e., not only the individuals analysed for their $\delta^{13} \mathrm{C}_{\mathrm{AA}}$ values for this study.
}

sheep/goat), pigs, dogs, marine mammals (grey seal, harp seal, harbor porpoise, ringed seal), saltwater fish (cod, herring), birds (black-throated diver), and pike, which all inhabit the brackish aquatic environment of the Baltic Sea. Stable isotopic results from bulk protein analysis suggested a significant contribution of marine protein to human $\operatorname{diet}\left(\delta^{13} \mathrm{C}-15\right.$ to $-13 \% ; ; \delta^{15} \mathrm{~N}+16$ to $+18 \%$ o).

During the late medieval period, the city of Visby on Gotland Island was under strong German influence as a member of the Hanseatic League, an economic trading alliance that included numerous cities on the northern coast of Europe. Bone and dentin samples from eleven humans recovered from the burial grounds of three parishes, St. Hans, St. Mikael, and Sta. Gertrud, are analyzed here. A variety of fauna, including dogs, cats, chicken, cattle, rats, and several species of fish (herring, pike, and roach) are also investigated. Previous analysis of bulk bone collagen isotopic compositions suggest that these humans had a mixed diet, including both marine and terrestrial inputs $\left(\delta^{13} \mathrm{C}-21\right.$ to $-18 \%$; $\delta^{15} \mathrm{~N}+9$ to $+15 \%$; Lindkvist 2008).

\section{Analytical methods}

Collagen was extracted from bone or tooth dentin powder at the Archaeological Research Laboratory, Stockholm University, using the method described in Brown et al. (1988). Collagen samples of $\sim 0.5 \mathrm{mg}$ were weighed into tin capsules and isotopic analysis was performed using a Carlo Erba NC2500 elemental analyzer coupled to a Finnigan MAT Delta $^{\text {Plus }}$ mass spectrometer via a split interface. Bulk collagen isotopic analyses were performed at the Stable Isotope Laboratory (SIL) in the Department of Geological Sciences, Stockholm University. The $\delta^{13} \mathrm{C}$ values were calibrated to VPDB using NBS-18, NBS-19, IAEA-CO-1 and IAEA-CO8 , and the $\delta^{15} \mathrm{~N}$ values were calibrated to AIR using IAEA-N1, IAEA-N2, IAEA-NO-3, and USGS25. Precision was monitored throughout using in-house standards (acetanilide and pepton) and was $\pm 0.1 \%$ o for both carbon- and nitrogenisotope analyses.

For each sample, approximately $2 \mathrm{mg}$ of collagen was hydrolyzed by heating at $110^{\circ} \mathrm{C}$ in $6 \mathrm{M} \mathrm{HCl}$ for $24 \mathrm{~h}$. Collagen hydrolysates of free amino acids were dried under $\mathrm{N}_{2}$ at $60{ }^{\circ} \mathrm{C}$ and redissolved in $1 \mathrm{ml}$ HPLC-grade water for LC/IRMS analysis. Amino acid $\delta^{13} \mathrm{C}$ values were obtained using a Surveyor HPLC connected to a Delta V Plus mass spectrometer via an LC Isolink interface (Thermo Scientific). Amino acids were resolved on a Primesep A column $(250 \mathrm{~mm} \times 3.2 \mathrm{~mm}, 5 \mu \mathrm{m}$ particle size, $100 \AA$ pore size, SiELC Technologies Ltd.) using a linear two-phase mobile phase gradient progressing from $100 \%$ water to $100 \%$ $0.024 \mathrm{M} \mathrm{H}_{2} \mathrm{SO}_{4}$ (Dunn et al. 2011; Honch et al. 2012; McCullagh et al. 2006; McCullagh 2010). Reference $\mathrm{CO}_{2}$ gas was calibrated against IAEA L-glutamic acid (USGS40; 
Fig. 1 Map of Sweden showing the locations of archaeological sites included in this study

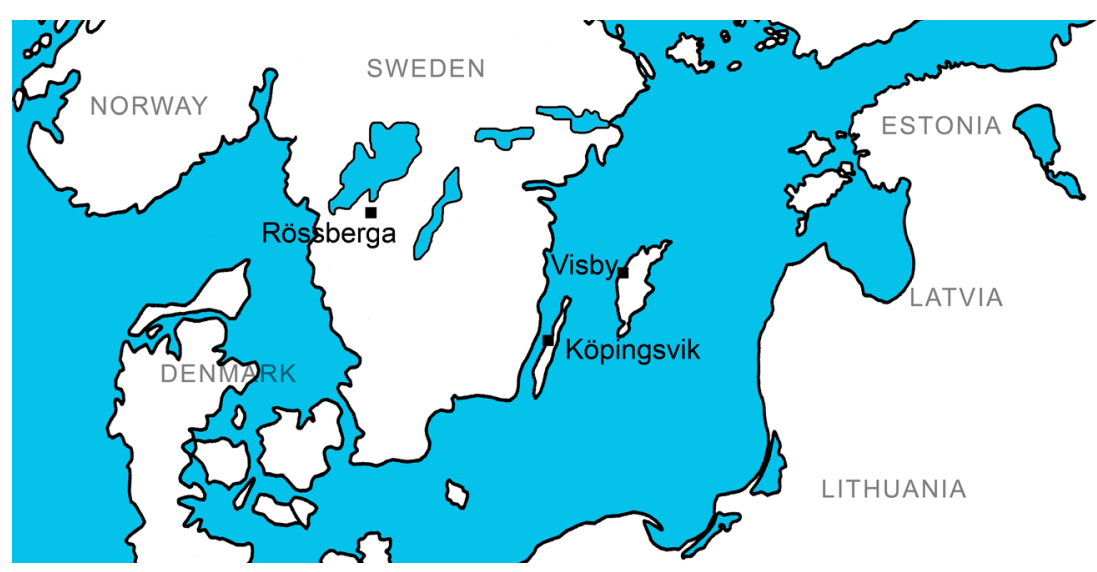

$\delta^{13} \mathrm{C}-26.2 \%$ ). Accuracy and reproducibility were monitored throughout using a standard amino acid mix incorporating a wide range of $\delta^{13} \mathrm{C}$ values $\left(\delta^{13} \mathrm{C}_{\mathrm{Asp}}-22.4 \%\right.$; $\delta{ }^{13} \mathrm{C}_{\mathrm{Glu}}$ $-26.2 \% ; \delta^{13} \mathrm{C}_{\mathrm{Gly}}-32.4 \% ; \delta \delta^{13} \mathrm{C}_{\mathrm{Ala}}-26.1 \%$; $\delta{ }^{13} \mathrm{C}_{\text {Pro }}$ $-10.6 \%$; Sigma-Aldrich). All archaeological samples were analyzed in duplicate. Data were analyzed with Isodat software (version 2.5). All analyte peaks were manually integrated to ensure that $\delta^{13} \mathrm{C}$ values were accurately reported.

\section{Results}

Bulk protein carbon- and nitrogen-isotope compositions are summarized in Fig. 2 and Table 2 (see also Eriksson et al. 2008; Linderholm 2008; Lindkvist 2008). For amino acid isotopic analyses, collagen preservation was assessed by comparing each sample amino acid distribution to the theoretical distribution for collagen. All reported amino acids were preserved within $10 \%$ of the theoretical value (Dunn 2011). The $\delta^{13} \mathrm{C}$ values are reported for the following amino acids: aspartic acid/asparagine, hydroxyproline, glutamatic acid/glutamine, threonine, serine, methionine, histidine, glycine, alanine, proline, valine, isoleucine and leucine, lysine, phenylalanine, and arginine. Isoleucine and leucine co-elute and are thus reported as a single value. Aspartic acid and asparagine are indistinguishable post-hydrolysis, as are glutamic acid and glutamine, and are reported as single values. Serine typically elutes in a region where the baseline drops with the introduction of the sulfuric acid component of the mobile phase, and the $\delta^{13} \mathrm{C}_{\mathrm{Ser}}$ values are less accurate because of the difficulty of integrating peaks in such as dynamic region. Low abundance amino acids (i.e., methionine and histidine) are likewise not considered interpretively useful because of poor reproducibility compared to the more abundant amino acids. All the $\delta^{13} \mathrm{C}_{\mathrm{AA}}$ values are reported in Table 2 and shown graphically in Fig. 3.

A summary of the isotopic compositions for four comparative human populations is presented in Table 3 to provide context for the amino acid and bulk protein isotopic compositions reported. The range for the $\delta^{13} \mathrm{C}_{\mathrm{NE}-\mathrm{AA}}$ values for humans excavated at Rössberga is $14.0 \%$ [ -27.5 to $-13.5 \%$ o and is $21.0 \%$ [ -30.6 to $-9.6 \%$ ] for essential amino acids. Similarly, the range in faunal $\delta^{13} \mathrm{C}_{\mathrm{NE}-\mathrm{AA}}$ values is $12.9 \%$ [ -26.6 to $-13.7 \%$ ] and for essential amino acids, the range in $\delta^{13} \mathrm{C}$ values is $15.7 \%$ [ $[-31.0$ to $-15.3 \%$ ]. The small ranges for Rössberga fauna compared to Köpingsvik and Visby (below) for both the $\delta^{13} \mathrm{C}_{\mathrm{E}-\mathrm{AA}}$ and $\delta^{13} \mathrm{C}_{\mathrm{E}-\mathrm{AA}}$ values likely result from the absence of marine or freshwater fauna among the specimens available for analysis from this site. The absolute $\delta^{13} \mathrm{C}$ values for Rössberga are likewise lower than those determined for the other two sites, again most likely due to predominately or exclusively terrestrial $\mathrm{C}_{3}$ plant-derived protein consumption by both humans and fauna. For the humans from Köpingsvik, the range in $\delta^{13} \mathrm{C}_{\mathrm{NE}-\mathrm{AA}}$ is $16.9 \%$ $[-20.0$ to $-3.1 \%$ o $]$ and the range in $\delta^{13} \mathrm{C}_{\mathrm{E}-\mathrm{AA}}$ values is $20.0 \%$ $\left[-25.3\right.$ to $-5.3 \%$ ]. The range of faunal $\delta^{13} \mathrm{C}_{\mathrm{NE}-\mathrm{AA}}$ values is somewhat larger $(26.8 \%$ [ $[-26.6$ to $+0.2 \% \circ])$, as is the range for essential amino acids $(25.2 \%$ o -30.6 to $-5.4 \%$ o $)$. The larger ranges for fauna compared to humans from the same site is expected, because the faunal specimens represent diverse habitats and dietary ecologies. Finally, for Visby, the range of human $\delta^{13} \mathrm{C}_{\mathrm{NE}-\mathrm{AA}}$ values was $15.0 \%$ [ $[-24.7$ to $-9.7 \%$ o $]$ and $19.5 \%$ [ -30.2 to $-10.7 \%$ o] for essential amino acids. For faunal specimens, the range of $\delta^{13} \mathrm{C}_{\mathrm{NE}-\mathrm{AA}}$ values was $25.0 \%$ [ -25.6 to $-0.6 \%$ ] and, for essential amino acids, the range was $22.8 \%$ [ -31.8 to $-9.0 \%$ ]. Again, the larger range of $\delta^{13} \mathrm{C}_{\mathrm{AA}}$ values for fauna relative to humans reflects greater habitat and dietary diversity among the different species analyzed.

\section{Discussion}

Comparative $\delta^{13} \mathrm{C}_{\mathrm{AA}}$ values from a variety of ecological and archaeological settings, some previously published, are used to contextualize the human isotopic results from this study. All the amino acid carbon-isotope compositions were generated using a similar laboratory 
Table 1 Description of archaeological human and faunal specimens from all sites

\begin{tabular}{|c|c|c|c|}
\hline Sample ID & Species & Element & Date $^{\mathrm{a}}$ \\
\hline \multicolumn{4}{|l|}{ Rössberga } \\
\hline ROSS05 & Human & Humerus & $4420 \pm 40$ \\
\hline ROSS06 & Human & Humerus & $4400 \pm 45$ \\
\hline ROSS08 & Human & Humerus & $4550 \pm 45$ \\
\hline ROSS09 & Human & Humerus & $4455 \pm 45$ \\
\hline ROSS10 & Human & Humerus & $4325 \pm 45$ \\
\hline ROSS13 & Human & Humerus & $4480 \pm 45$ \\
\hline ROSS15 & Human & Humerus & $4790 \pm 50$ \\
\hline ROSS16 & Human & Humerus & $4890 \pm 50$ \\
\hline ROSS18 & Human & Humerus & $4685 \pm 50$ \\
\hline ROSS20 & Human & Humerus & $4780 \pm 45$ \\
\hline ROSS33 & Cow & Molar tooth & $4090 \pm 40$ \\
\hline ROSS35 & Dog & Femur & $4530 \pm 60$ \\
\hline ROSS36 & Hare & Tibia & $3395 \pm 55$ \\
\hline ROSS32 & Pig & Phalanx & $4360 \pm 45$ \\
\hline ROSS34 & Fox & Tibia & $3785 \pm 45$ \\
\hline \multicolumn{4}{|c|}{ Köpingsvik ${ }^{\mathrm{b}}$} \\
\hline KOP09 & Human & First molar & $4645 \pm 45$ \\
\hline KOP18 & Human & Mandible & $5580 \pm 60$ \\
\hline KOP30 & Human & Third molar & $4385 \pm 40$ \\
\hline KOP32 & Human & First molar & $4395 \pm 40$ \\
\hline KOP55 & Human & Mandible & $4200 \pm 45$ \\
\hline KOP70 & Human & Cranium & $4475 \pm 50$ \\
\hline KOP110 & Human & Ulna & $4290 \pm 40$ \\
\hline KOP112 & Human & Ulna & $4350 \pm 40$ \\
\hline KOP120 & Human & Fibula & $4385 \pm 40$ \\
\hline KOP129 & Grey seal & Zygomatic bone & \\
\hline КОР97 & Harp seal & Temporal bone & \\
\hline KOP98 & Harp seal & Temporal bone & \\
\hline KOP166 & Harbour porpoise & Vertebra & \\
\hline KOP167 & Harbour porpoise & Vertebra & \\
\hline KOP180 & Ringed seal & Humerus & \\
\hline KOP142 & Moose & Antler & \\
\hline KOP162 & Moose & Antler & \\
\hline KOP164 & Cow & Talus & $4150 \pm 48$ \\
\hline KOP182 & Cow & Humerus & \\
\hline KOP183 & Sheep/goat & Tooth & \\
\hline KOP149 & Pig & Tooth & \\
\hline KOP150 & Pig & Humerus & \\
\hline KOP151 & Pig & Molar & \\
\hline KOP156 & Pig & Calcaneus & \\
\hline KOP174 & Pig & Molar & \\
\hline KOP106 & Dog & Humerus & \\
\hline KOP145 & Dog & Humerus & \\
\hline KOP173 & Dog & Radius & \\
\hline KOP176 & Dog & Tooth & \\
\hline KOP178 & Dog & Temporal bone & \\
\hline KOP124 & Black-throated diver & Tarsometatarsus & \\
\hline
\end{tabular}

Table 1 (continued)

\begin{tabular}{|c|c|c|c|}
\hline Sample ID & Species & Element & Date $^{\mathrm{a}}$ \\
\hline KOP138 & Herring & Vertebra & \\
\hline KOP139 & Pike & Vertebra & \\
\hline KOP141 & Pike & Vertebra & \\
\hline KOP134 & Cod & Vertebra & \\
\hline KOP175 & Cod & Vertebra & \\
\hline \multicolumn{4}{|l|}{ Visby $^{\mathrm{c}}$} \\
\hline STH25 & Human & Second molar & Late Medieval \\
\hline STH31 & Human & Second molar & Late Medieval \\
\hline STH27 & Human & Second molar & Late Medieval \\
\hline STH18 & Human & Humerus & Late Medieval \\
\hline STH17 & Human & Femur & Late Medieval \\
\hline SMI14 & Human & Second molar & Late Medieval \\
\hline STM05 & Human & Femur & Late Medieval \\
\hline STM08 & Human & Femur & Late Medieval \\
\hline STM03 & Human & Mandible & Late Medieval \\
\hline STG03 & Human & Femur & Late Medieval \\
\hline STG05 & Human & Femur & Late Medieval \\
\hline DBV09 & Cow & & Late Medieval \\
\hline DBV11 & Cow & & Late Medieval \\
\hline DBV10 & Cow & & Late Medieval \\
\hline DBV26 & Dog & & Late Medieval \\
\hline DBV23 & Cat & & Late Medieval \\
\hline DBV25 & Cat & & Late Medieval \\
\hline DBV24 & Cat & & Late Medieval \\
\hline DBV19 & Rat & & Late Medieval \\
\hline DBV17 & Rat & & Late Medieval \\
\hline DBV20 & Rat & & Late Medieval \\
\hline DBV30 & Chicken & & Late Medieval \\
\hline DBV31 & Chicken & & Late Medieval \\
\hline DBV02 & Herring & & Late Medieval \\
\hline DBV03 & Pike & & Late Medieval \\
\hline DBV04 & Cod & & Late Medieval \\
\hline DBV01 & Roach & & Late Medieval \\
\hline
\end{tabular}

${ }^{\mathrm{a}} \mathrm{AMS}{ }^{14} \mathrm{C}$ dates reported in uncalibrated years $\mathrm{BP}$ as available; Late Medieval coincides with the 13th to 15th centuries

${ }^{\mathrm{b}}$ For Köpingsvik samples, AMS ${ }^{14} \mathrm{C}$ dates are reported in Linderholm et al. (2014)

${ }^{\mathrm{c}}$ For Visby samples, different prefixes denote cemetery, where STH indicates St. Hans, SMI/STM indicate St. Mikael, and STG indicates Sta. Gertrude

methodology (Honch et al. 2012; see also Supplementary Table 1). Briefly, archaeological human $\delta^{13} \mathrm{C}_{\mathrm{AA}}$ values representing high marine protein consumption (Japan and Greenland), terrestrial $\mathrm{C}_{4}$-derived protein consumption (Mesoamerica), high freshwater protein consumption (Iron Gates Region), and high $\mathrm{C}_{3}$-derived protein consumption (Eastern Europe) are used to assess the efficacy of the proxies tested here in distinguishing among 


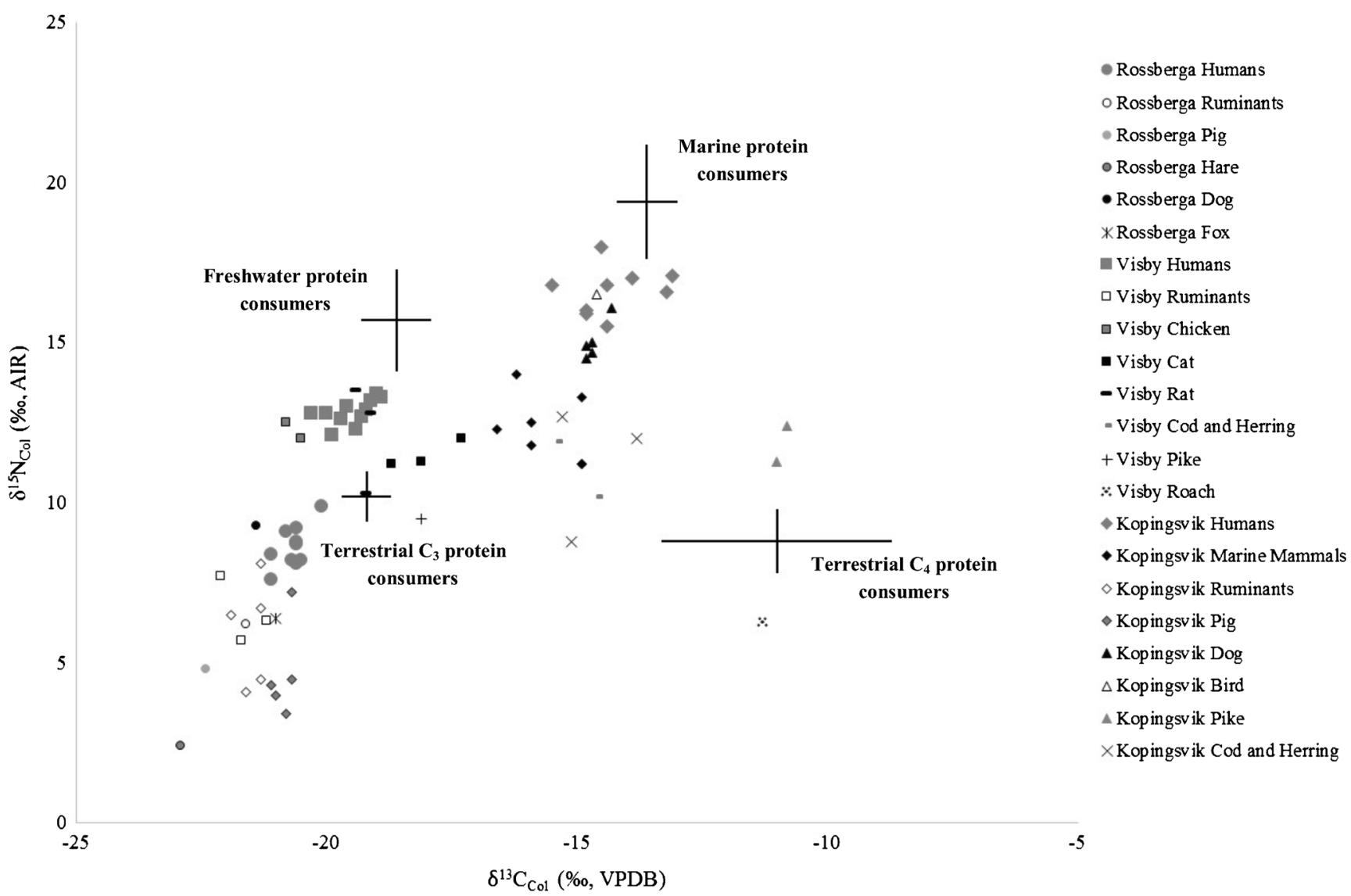

Fig. 2 The $\delta^{13} \mathrm{C}_{\mathrm{col}}$ and $\delta^{15} \mathrm{~N}_{\mathrm{col}}$ values for human and faunal specimens included in this study

different dietary regimes. Although there is necessarily some degree of uncertainty in all palaeodietary reconstructions, the interpretation of the dietary isotopic data for these four well-constrained groups is supported by archaeological and zooarchaeological evidence.

\section{Terrestrial vs. aquatic resource consumption}

The $\Delta^{13} \mathrm{C}_{\text {Gly-Phe }}$ proxy effectively discriminates between diets dominated by aquatic (marine and freshwater) and terrestrial $\left(\mathrm{C}_{3}\right.$ and $\mathrm{C}_{4}$ plant-derived) protein, and this has been demonstrated in archaeological human and faunal specimens (Choy et al. 2010; Corr et al. 2005). Phenylalanine is an essential amino acid that has been shown to be directly routed from diet to tissue with very little isotopic fractionation $(\sim 1-2 \%$ ) , based on archaeological data from Honch et al. (2012) and other published datasets (e.g., Choy et al. 2010; Corr et al. 2005), as well as controlled feeding studies using mice (Jones 2002) and pigs (Howland et al. 2003). In contrast, the isotopic composition of glycine, a nonessential amino acid, varies systematically between terrestrial and marine ecosystems, where marine glycine $\delta^{13} \mathrm{C}$ values tend to be high. Terrestrial protein consumers are generally expected to have lower $\Delta^{13} \mathrm{C}_{\text {Gly-Phe }}$ values than aquatic protein consumers from the same environment or context. Aquatic protein consumers typically have $\Delta^{13} \mathrm{C}_{\mathrm{Gly}}$-Phe values greater than $15 \% o^{2}$, and terrestrial protein consumers have $\Delta^{13} \mathrm{C}_{\mathrm{Gly}-\mathrm{Phe}}$ values $\sim 10-12 \%$ o or lower (i.e., for terrestrial herbivores). The $\Delta^{13} \mathrm{C}_{\mathrm{Gly}}$-Phe values for Rössberga human specimens are similar to the average $\Delta^{13} \mathrm{C}_{\mathrm{Gly}}$-Phe values for the terrestrial plant-derived protein consumers (Fig. $4 \mathrm{a}, \mathrm{b}$ ). The average $\Delta^{13} \mathrm{C}_{\mathrm{Gly}}$-Phe value for the Rössberga humans was $+12.4 \pm 1.0$, and faunal values are similar, ranging from 8.2 (ROSS33, a cow) to 13.3 (ROSS35, a dog; Fig. 4a, b). Humans from Köpingsvik displayed higher $\Delta^{13} \mathrm{C}_{\text {Gly-Phe }}$ values $(+19.8 \pm 1.0)$ consistent with the greater consumption of marine resources inferred from the bulk carbon- and nitrogen-isotope compositions (Figs. 4 and 5). High average $\Delta^{13} \mathrm{C}_{\mathrm{Gly}-\mathrm{Phe}}$ values were exhibited by marine mammals $(+18.8 \pm 1.3)$, fish $(+18.6 \pm 2.5)$, and dogs $(+19.7$ \pm 0.9 ). For humans and these fauna, the high $\Delta^{13} \mathrm{C}_{\mathrm{Gly}-\mathrm{Phe}}$ values are largely a result of high $\delta^{13} \mathrm{C}_{\mathrm{Gly}}$ values rather than low $\delta^{13} \mathrm{C}_{\mathrm{Phe}}$ values. The relationship between high $\delta^{13} \mathrm{C}_{\mathrm{Gly}}$ values and marine protein consumption observed here is consistent with the findings of other archaeological studies (Corr et al. 2005; Hare et al. 1991; Honch et al. 2012). Herring, although an aquatic species, has a moderate $\Delta^{13} \mathrm{C}_{\text {Gly-Phe value }}$ $(+14.8)$. This apparently terrestrial value may reflect its relative low trophic position and dietary reliance on zooplankton,

\footnotetext{
${ }^{2}$ For samples using LC/IRMS instrumentation.
} 


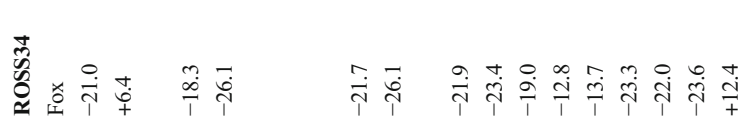

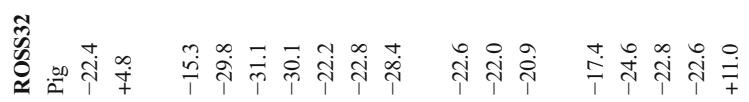
कृ

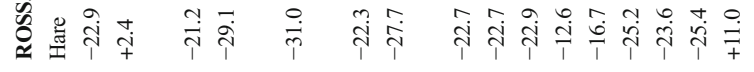

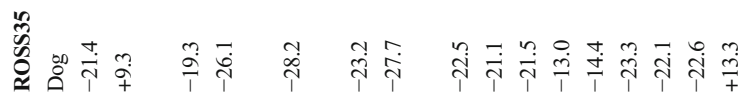

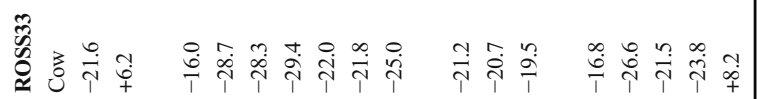

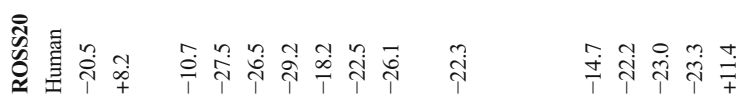

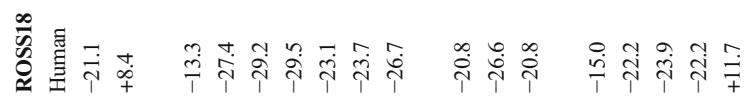

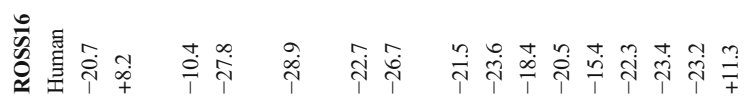
की

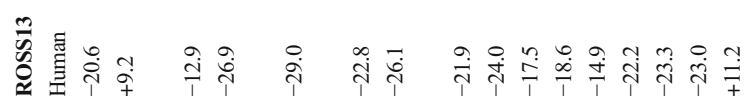

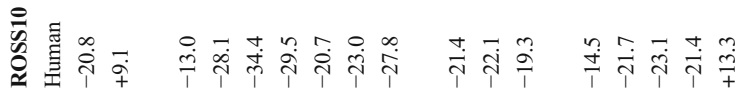

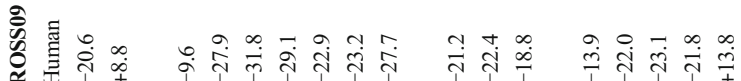

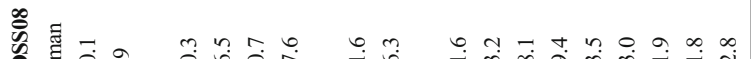

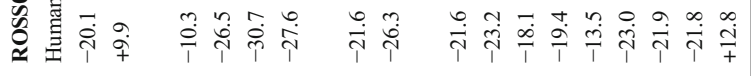

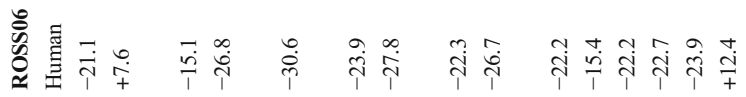

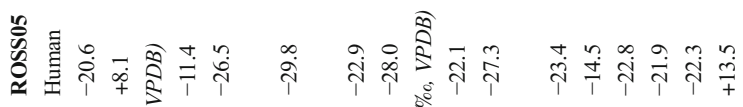

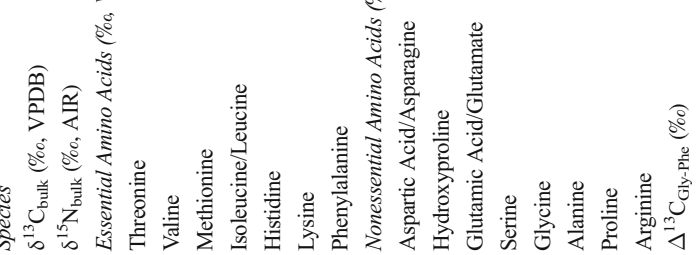

ta

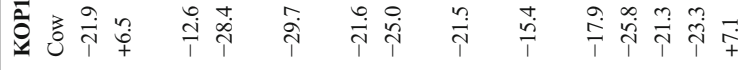

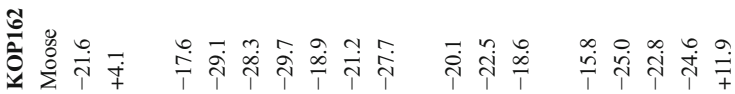

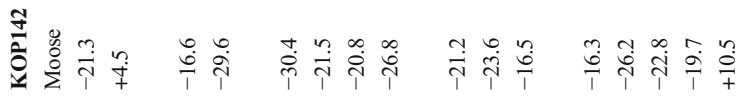

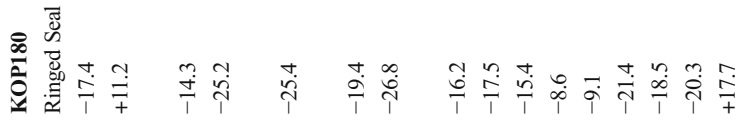

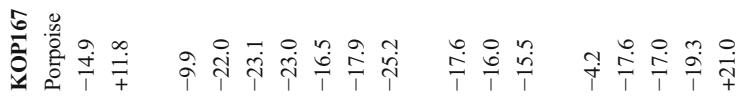

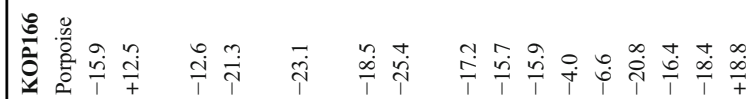

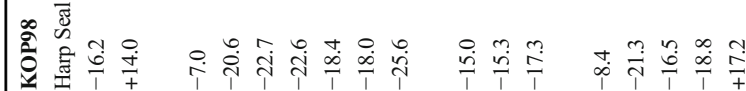

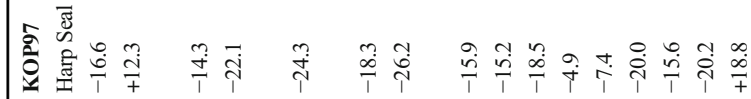

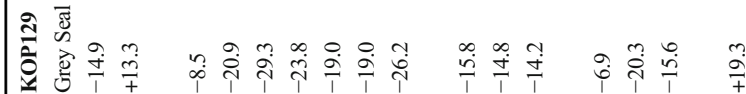

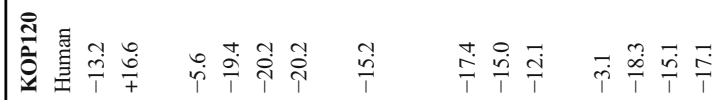

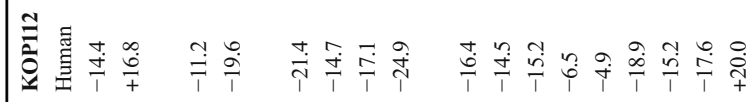

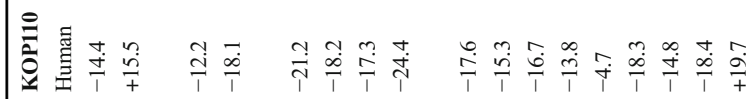

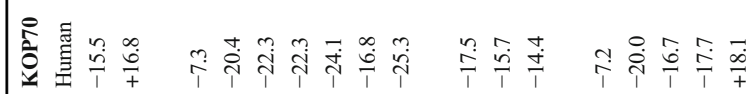

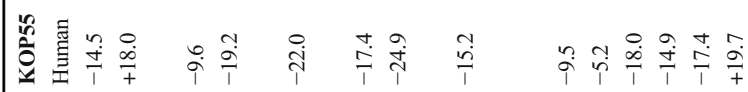

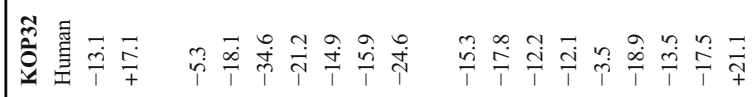

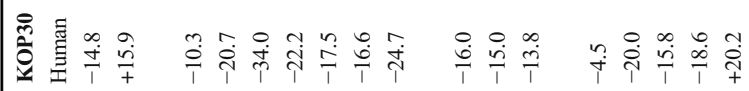

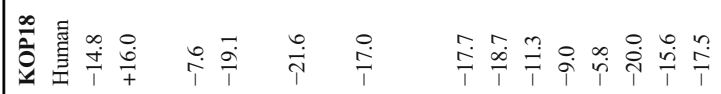

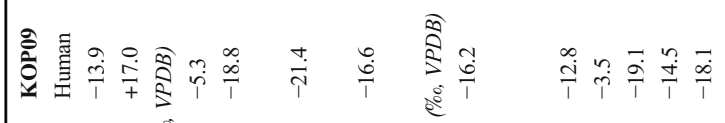

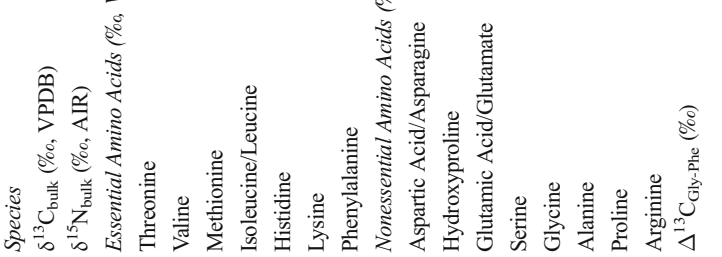




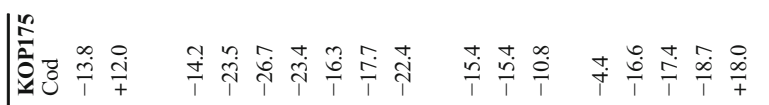

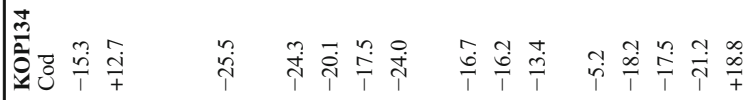

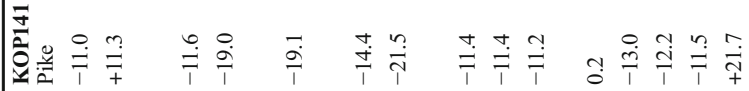

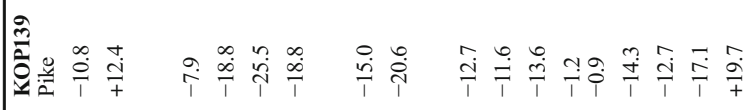

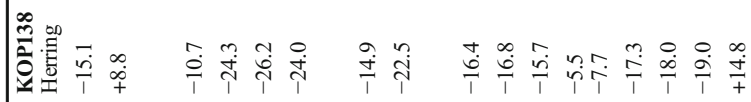

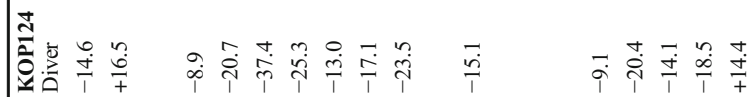

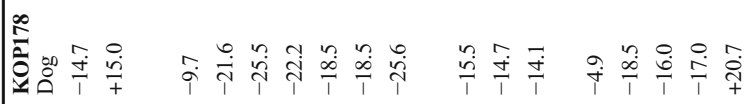

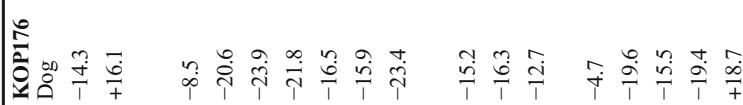

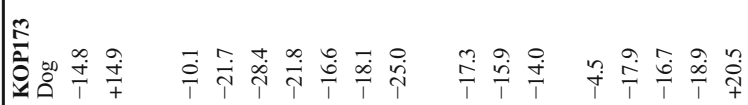

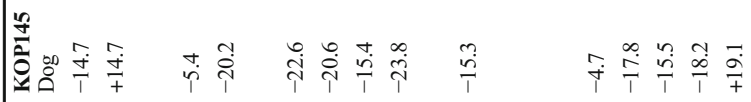

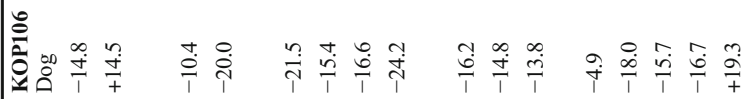

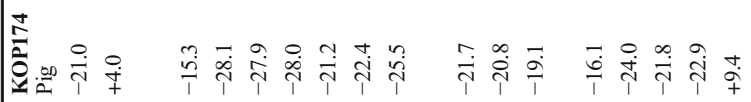

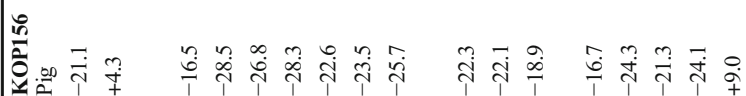

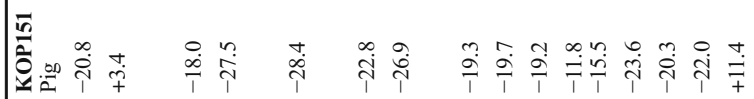

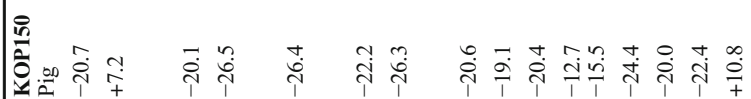

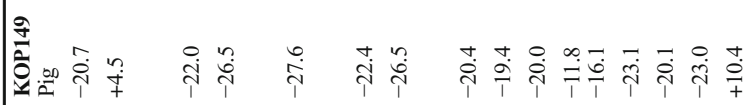

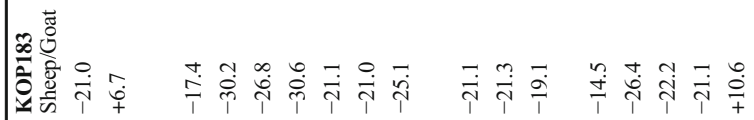

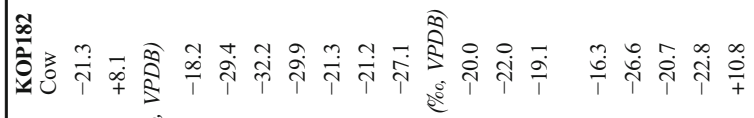
㩊离

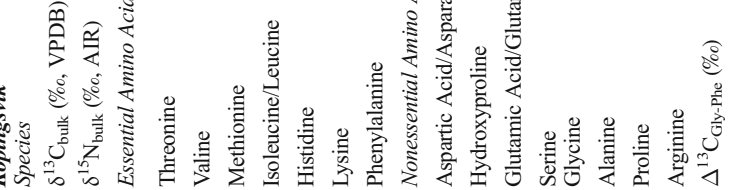

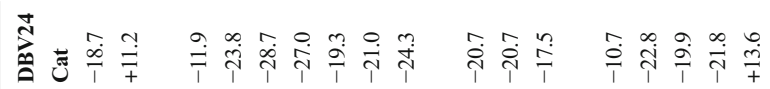

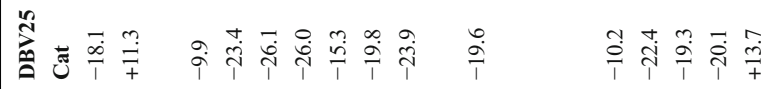

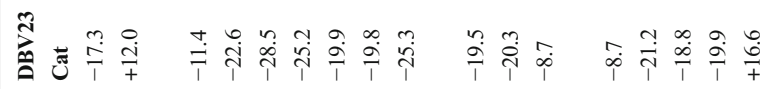

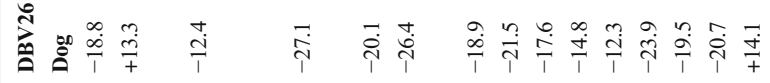

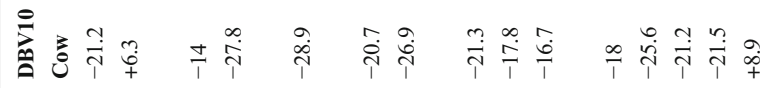

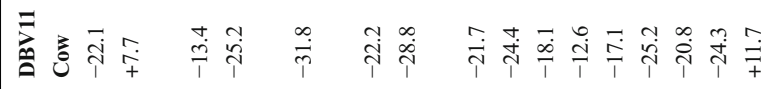

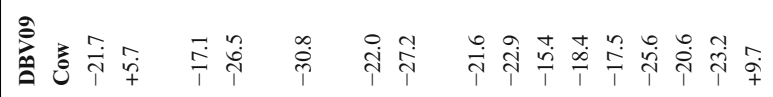

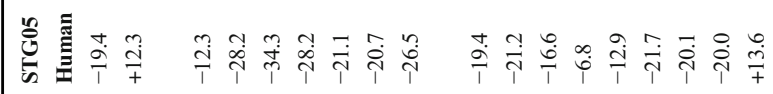

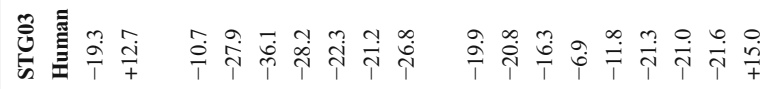

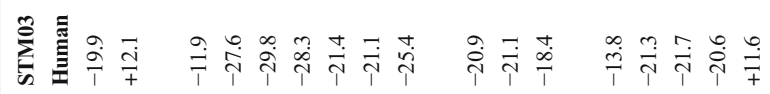

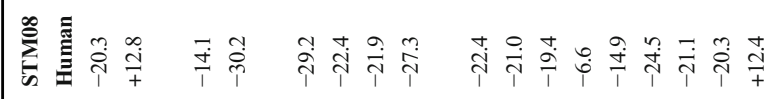

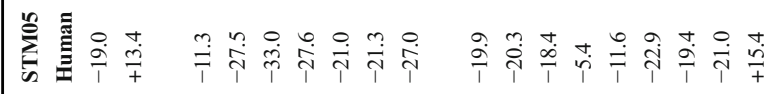

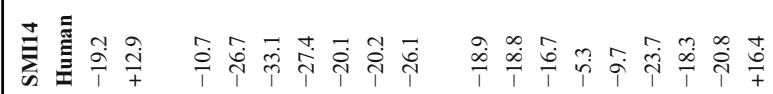

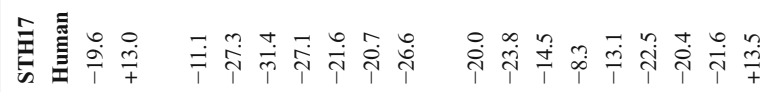

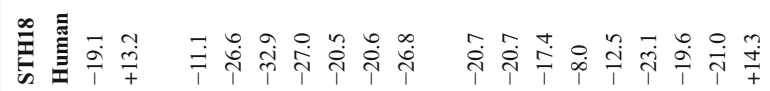

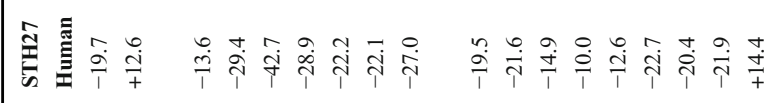

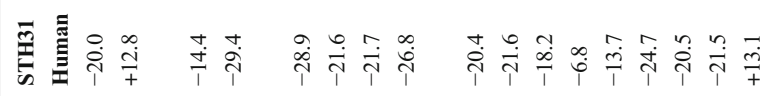

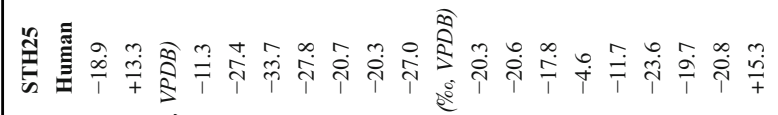

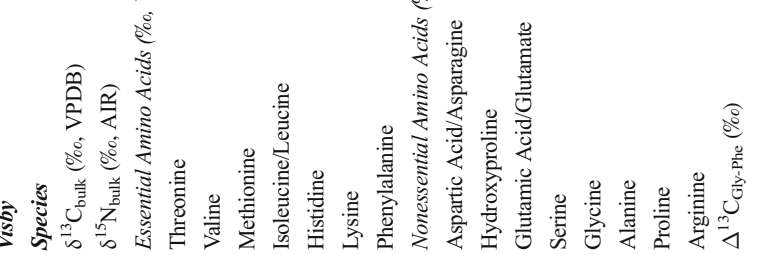


which has relatively low $\Delta^{13} \mathrm{C}_{\text {Gly-Phe }}$ values of approximately +9 (Dunn 2011). The $\Delta{ }^{13} \mathrm{C}_{\mathrm{Gly}}$-Phe values are comparatively low for terrestrial ruminants $(+10.2 \pm 1.8)$ and pigs $(+10.2 \pm 1.0)$, which is consistent with consumption of terrestrial protein.

Using bulk carbon- and nitrogen-isotope compositions, a mixed diet incorporating both terrestrial and marine protein was inferred for humans from Visby. Human $\Delta^{13} \mathrm{C}_{\text {Gly-Phe }}$ values are intermediate relative to Rössberga and Köpingsvik specimens, with an average $\Delta^{13} \mathrm{C}_{\mathrm{Gly}}$-Phe value of $+14.1 \pm 1.4$. For fish, the average $\Delta^{13} \mathrm{C}_{\mathrm{Gly}}$-Phe value is high $(+19.1 \pm 0.8)$, again driven by the high $\delta^{13} \mathrm{C}_{\mathrm{Gly}}$ values. Terrestrial fauna (cattle, dogs, cats, rats, and chickens) have moderate $\Delta{ }^{13} \mathrm{C}_{\text {Gly-Phe values }(+12.9}$ \pm 2.3 ), reflecting a predominately terrestrial diet for these animals. The higher $\Delta^{13} \mathrm{C}_{\mathrm{Gly} \text {-Phe }}$ value determined for one of the cats (DBV23; +16.6) suggests consumption of aquatic protein, perhaps through scavenging or being fed by humans. On the whole, the $\Delta^{13} \mathrm{C}_{\mathrm{Gly}}$-Phe values for Visby humans and fauna support the inference of a mixed terrestrial-aquatic diet, with perhaps greater emphasis on terrestrial resources (Figs. 4 and 5).

\section{Marine vs. freshwater resource consumption}

The bivariate distribution of $\delta^{13} \mathrm{C}_{\mathrm{Val}}$ and $\delta^{13} \mathrm{C}_{\text {Phe }}$ values shows potential for distinguishing between marine and freshwater resource consumption. Valine, although its recovery must be monitored closely during analysis, was determined by Honch et al. (2012) to vary in a fairly systematic fashion between marine/ $\mathrm{C}_{4}$ protein consumers and freshwater $/ \mathrm{C}_{3}$ protein consumers, and is present in protein-rich foods, such as terrestrial meat, soy and other legumes, and fish. Although there is no archaeological or zooarchaeological evidence to suggest freshwater resource exploitation, the $\delta^{13} \mathrm{C}_{\mathrm{Val}}$ and $\delta^{13} \mathrm{C}_{\mathrm{Phe}}$ values are applied here to test this assumption for humans from the three sites (Fig. 6). The Rössberga humans cluster close to the $C_{3}$ plant-derived terrestrial protein consumer, exhibiting low $\delta^{13} \mathrm{C}_{\mathrm{Val}}$ values and moderately low $\delta^{13} \mathrm{C}_{\mathrm{Phe}}$ values. In contrast, the Köpingsvik humans have higher $\delta^{13} \mathrm{C}_{\mathrm{Val}}$ values and somewhat high $\delta^{13} \mathrm{C}_{\text {Phe }}$ values, consistent with high marine protein consumption and excluding significant freshwater protein consumption. There is more variability among humans from Visby compared to the Rössberga humans, suggesting greater dietary diversity at the individual level, but the $\delta^{13} \mathrm{C}_{\text {Val }}-\delta^{13} \mathrm{C}_{\text {Phe }}$ plot does not indicate significant marine or freshwater protein consumption. Instead, a predominately $\mathrm{C}_{3}$ plant-derived protein source is indicated, although it must be noted that this relationship does not always differentiate well between $\mathrm{C}_{3}$ plantderived and freshwater protein consumption, or between $\mathrm{C}_{4}$ plant-derived and marine protein consumption.

\section{Relationship between $\delta^{15} \mathbf{N}_{\text {col }}$ and $\delta^{13} C_{P h e}$ values}

The bivariate plot of $\delta^{15} \mathrm{~N}_{\text {col }}$ and $\delta{ }^{13} \mathrm{C}_{\text {Phe }}$ values presents the relationship between the trophic level of consumed protein 
Table 3 Summary of comparative human $\delta^{13} \mathrm{C}_{\mathrm{AA}}, \delta^{13} \mathrm{C}_{\mathrm{col}}$, and $\delta^{15} \mathrm{~N}_{\mathrm{col}}$ values

\begin{tabular}{|c|c|c|c|c|}
\hline & $\begin{array}{l}\text { High marine } \\
\text { protein consumers }\end{array}$ & $\begin{array}{l}\text { High freshwater } \\
\text { protein consumers }\end{array}$ & $\begin{array}{l}\text { High } \mathrm{C}_{3} \text { terrestrial } \\
\text { protein consumers }\end{array}$ & $\begin{array}{l}\text { High } \mathrm{C}_{4} \text { terrestrial } \\
\text { protein consumers }\end{array}$ \\
\hline \multicolumn{5}{|c|}{ Amino acid isotopic compositions } \\
\hline Valine & $-17.9 \pm 1.5$ & $-24.2 \pm 2.0$ & $-26.3 \pm 0.8$ & $-19.1 \pm 2.3$ \\
\hline Lysine & $-15.6 \pm 0.4$ & $-22.6 \pm 0.5$ & $-22.5 \pm 1.3$ & $-16.5 \pm 1.7$ \\
\hline Phenylalanine & $-23.7 \pm 1.5$ & $-30.4 \pm 1.1$ & $-27.1 \pm 0.7$ & $-19.2 \pm 1.9$ \\
\hline Aspartic acid/asparagine & $-14.5 \pm 3.0$ & $-22.1 \pm 1.8$ & $-20.6 \pm 0.7$ & $-13.7 \pm 2.8$ \\
\hline Hydroxyproline & $-14.5 \pm 0.4$ & $-19.7 \pm 1.2$ & $-21.2 \pm 0.8$ & $-10.5 \pm 2.1$ \\
\hline Glutamic acid/glutamate & $-14.1 \pm 1.1$ & $-20.4 \pm 2.3$ & $-18.4 \pm 0.6$ & $-10.0 \pm 2.7$ \\
\hline Glycine & $-7.0 \pm 1.3$ & $-10.8 \pm 1.3$ & $-12.9 \pm 0.6$ & $-7.9 \pm 2.6$ \\
\hline Alanine & $-18.1 \pm 1.3$ & $-25.2 \pm 0.9$ & $-21.3 \pm 1.1$ & $-12.9 \pm 3.0$ \\
\hline Proline & $-13.8 \pm 0.4$ & $-20.3 \pm 1.0$ & $-21.5 \pm 0.9$ & $-11.4 \pm 2.3$ \\
\hline Arginine & $-17.4 \pm 0.8$ & $-23.0 \pm 1.0$ & $-22.6 \pm 1.5$ & $-13.9 \pm 1.9$ \\
\hline \multicolumn{5}{|l|}{ Bulk isotopic compositions } \\
\hline$\delta^{15} \mathrm{~N}_{\mathrm{col}}$ & $+19.4 \pm 1.8$ & $+15.7 \pm 1.6$ & $+10.2 \pm 0.8$ & $+8.8 \pm 1.0$ \\
\hline$\delta^{13} \mathrm{C}_{\mathrm{col}}$ & $-13.6 \pm 0.6$ & $-18.6 \pm 0.7$ & $-19.2 \pm 0.5$ & $-11.0 \pm 2.3$ \\
\hline
\end{tabular}

Data from Honch et al. (2012); all values are reported as an average \pm one standard deviation in \%o

and phenylalanine, the carbon-isotope composition of which is highly conserved between producers and consumers (Fig. 7). The human nitrogen isotopic compositions from Rössberga are

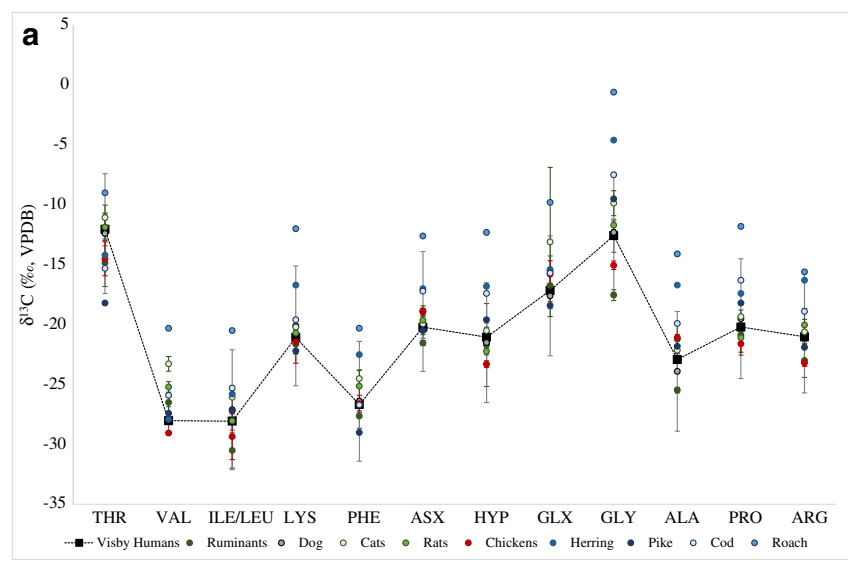

low $(<+10 \%$ ) compared to the other two sites, whereas the $\delta^{13} \mathrm{C}_{\mathrm{Phe}}$ values are moderately low and similar to the $\delta{ }^{13} \mathrm{C}_{\mathrm{Phe}}$ values for humans from Visby. The Visby humans do, however,

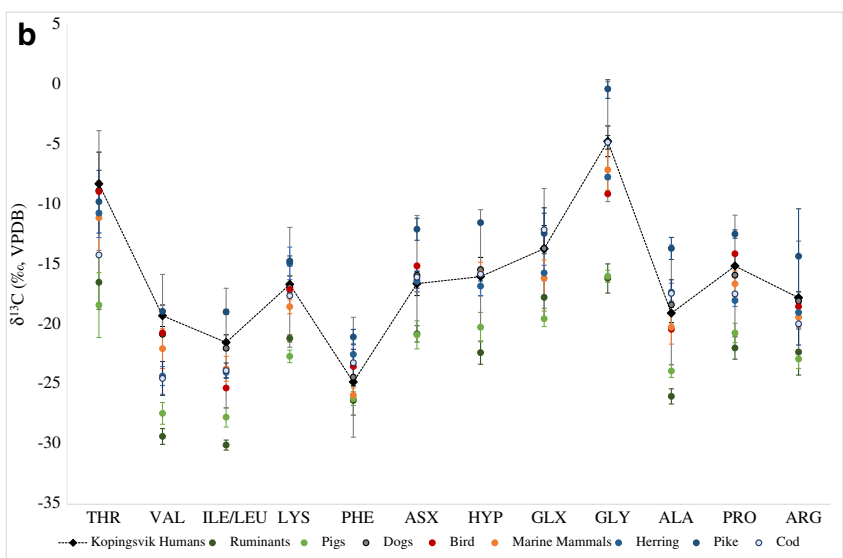

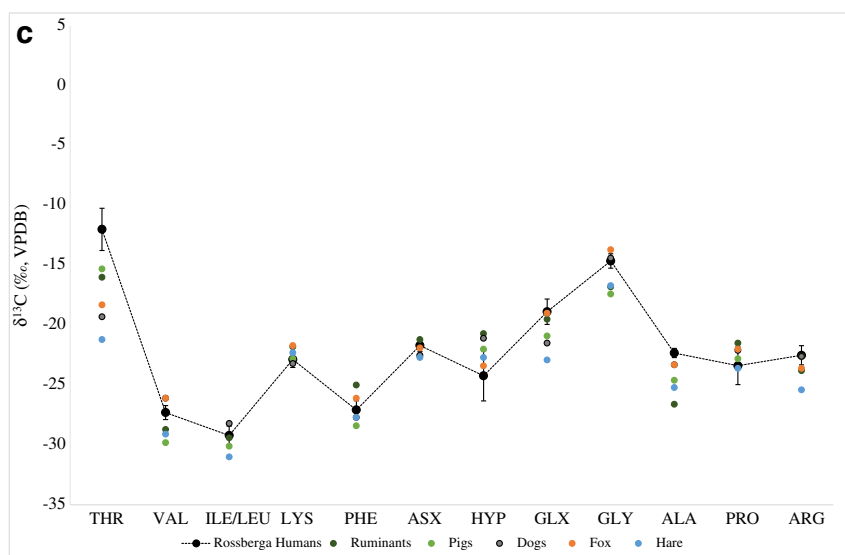

Fig. 3 Average essential and nonessential amino acid carbon-isotope compositions for a Visby, b Köpingsvik, and $\mathbf{c}$ Rössberga. Error bars represent \pm one standard deviation when $n>1$ 

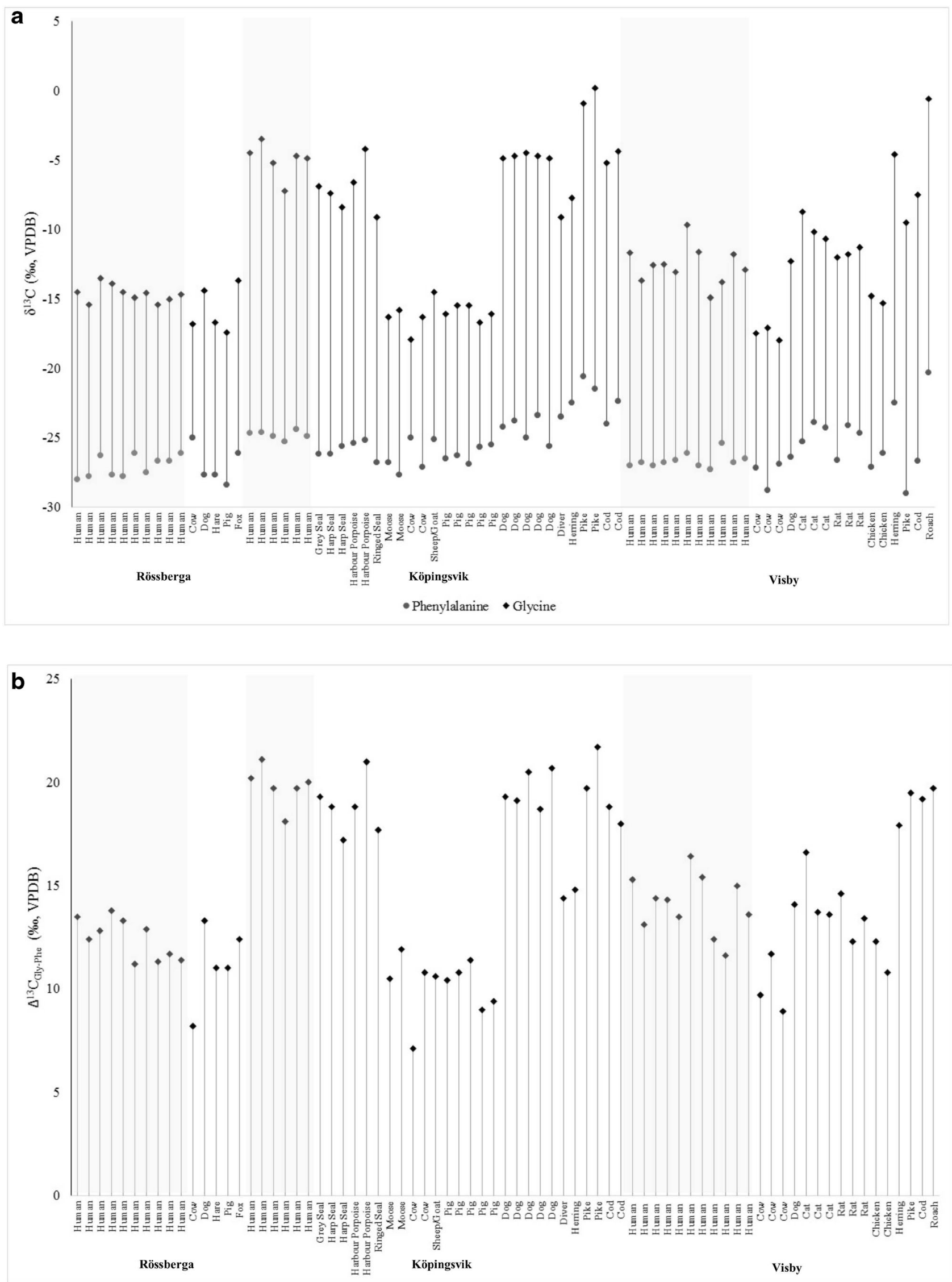

Fig. 4 a The $\delta^{13} \mathrm{C}_{\mathrm{Gly}}$ and $\delta^{13} \mathrm{C}_{\mathrm{Phe}}$ values for all humans and fauna. $\mathbf{b}$ The $\Delta{ }^{13} \mathrm{C}_{\mathrm{Gly} \text {-Phe }}$ proxy for all humans and fauna 


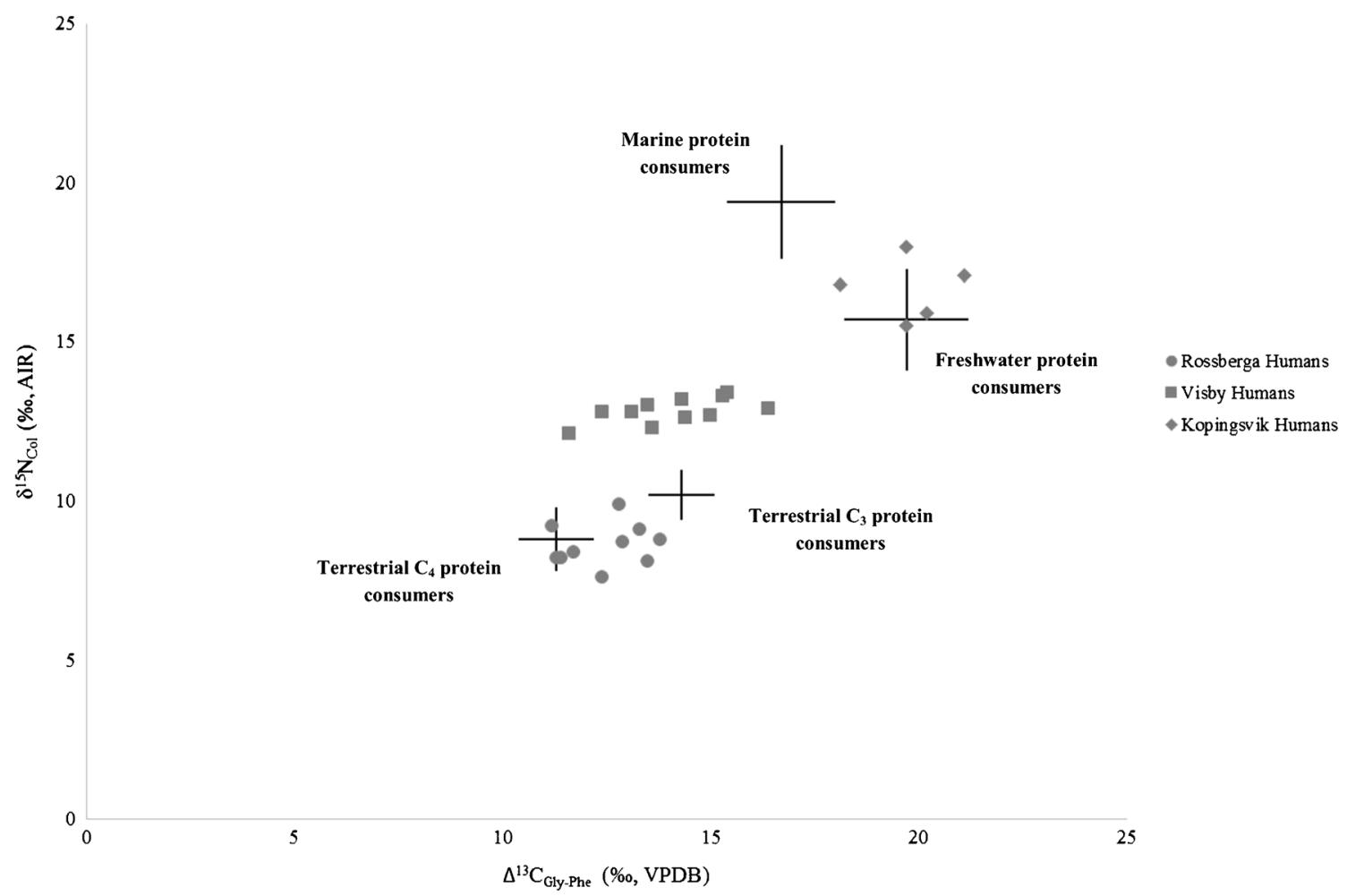

Fig. 5 The $\Delta^{13} \mathrm{C}_{\mathrm{Gly}-\mathrm{Phe}}$ proxy plotted with $\delta^{15} \mathrm{~N}_{\text {col }}$ values for all humans. The $\Delta^{13} \mathrm{C}_{\mathrm{Gly}-\mathrm{Phe}}$ and $\delta^{15} \mathrm{~N}_{\mathrm{col}}$ values for other archaeological humans (Honch et al. 2012) are plotted as average \pm one standard deviation

exhibit moderately higher $\delta^{15} \mathrm{~N}_{\text {col }}$ values (approximately +12.0 to $+13.5 \%$ ) , suggesting consumption of dietary protein from a trophic level higher than at Rössberga. Finally, the humans from Köpingsvik displayed higher $\delta^{15} \mathrm{~N}_{\text {col }}$ values (approximately

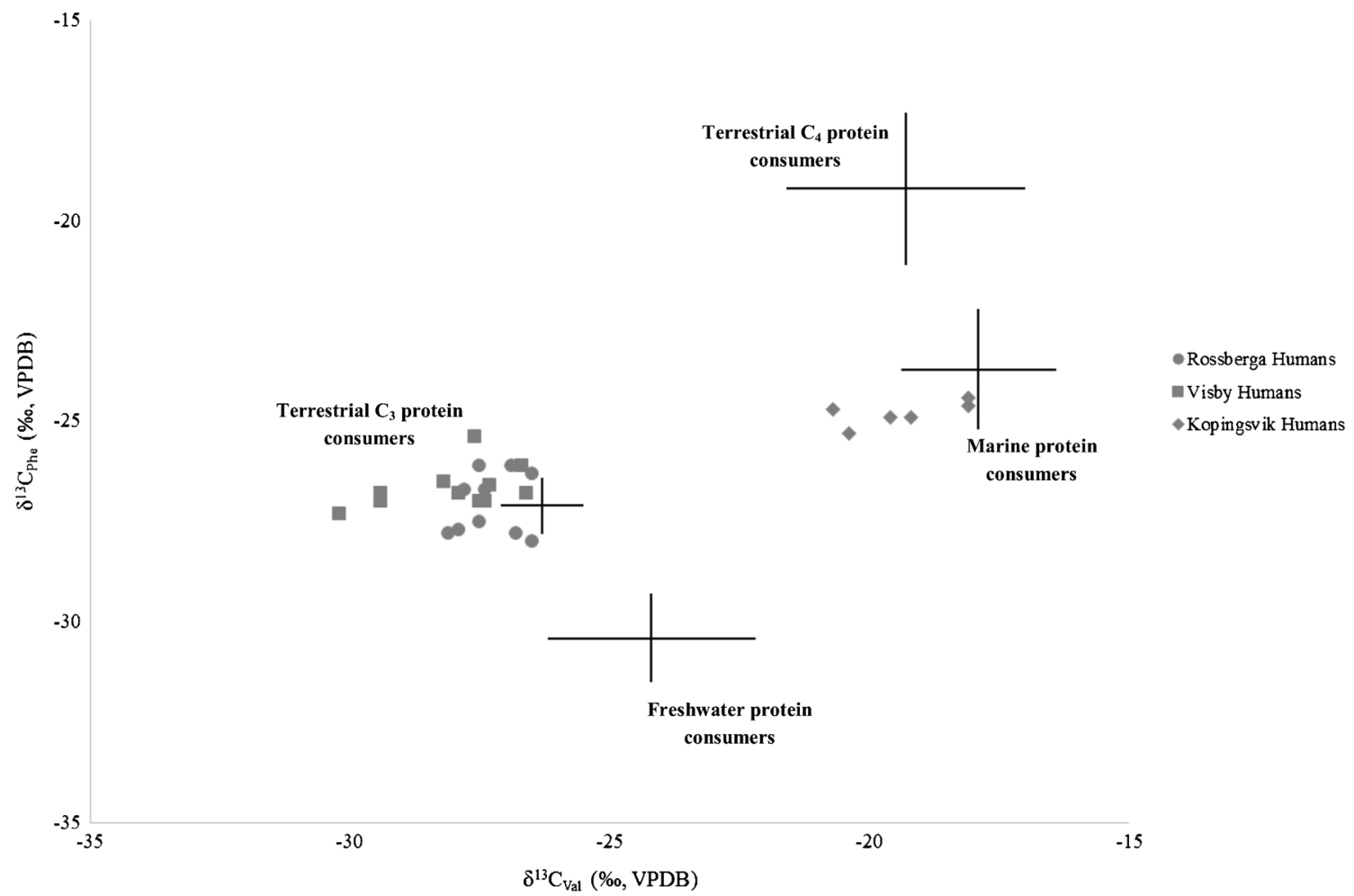

Fig. 6 The $\delta^{13} \mathrm{C}_{\text {Val }}$ vs. $\delta^{13} \mathrm{C}_{\text {Phe }}$ values for all humans. The $\delta{ }^{13} \mathrm{C}_{\mathrm{Val}}$ and $\delta^{13} \mathrm{C}_{\mathrm{Phe}}$ values for other archaeological humans (Honch et al. 2012) are plotted as average \pm one standard deviation 


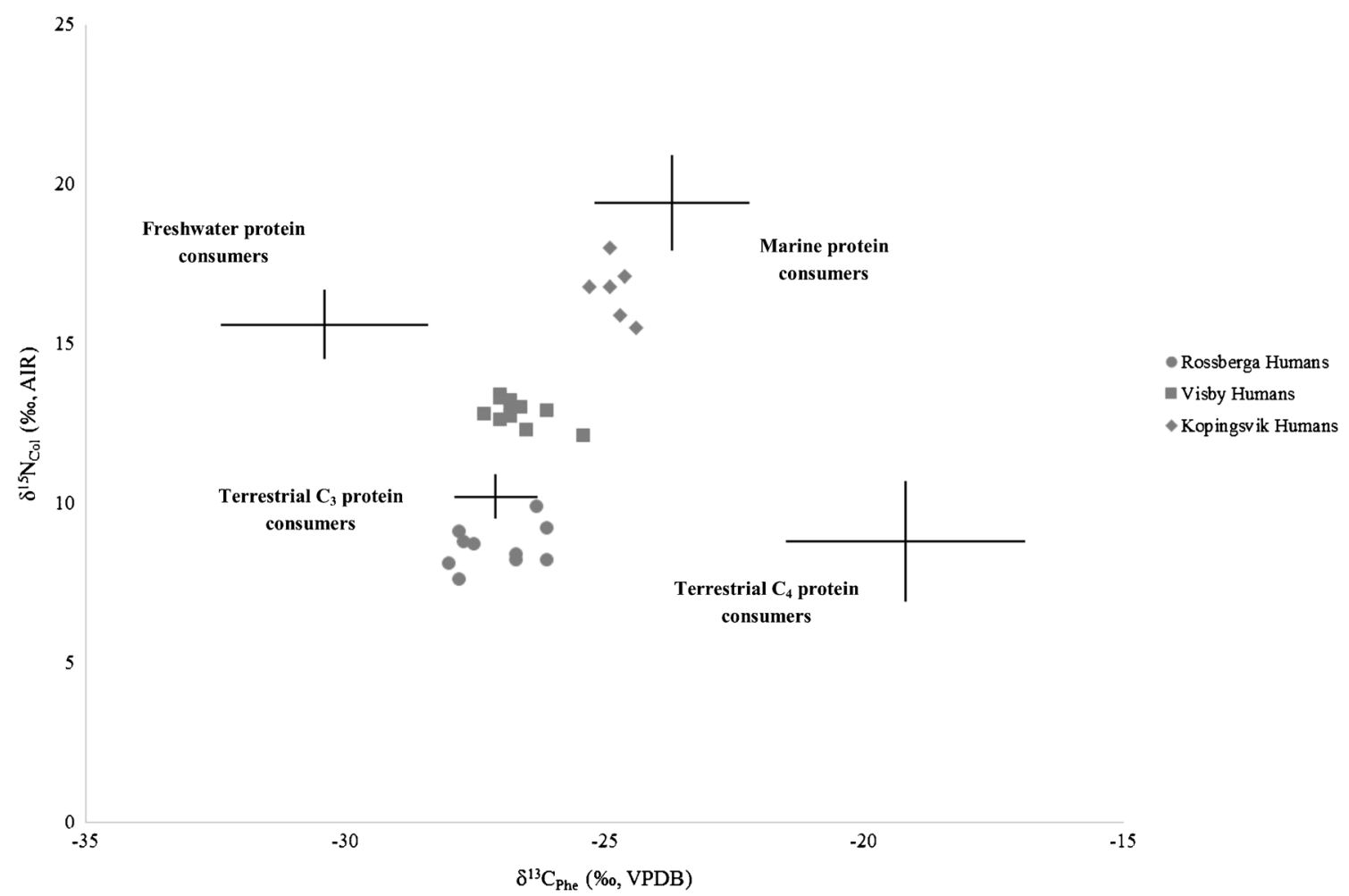

Fig. 7 The $\delta{ }^{13} \mathrm{C}_{\text {Phe }}$ vs. $\delta{ }^{15} \mathrm{~N}_{\text {col }}$ values for all humans. The $\delta{ }^{13} \mathrm{C}_{\text {Phe }}$ and $\delta{ }^{15} \mathrm{~N}_{\mathrm{col}}$ values for other archaeological humans (Honch et al. 2012) are plotted as an average \pm one standard deviation

+15.5 to $+18.0 \%$ ) and moderately higher $\delta^{13} \mathrm{C}_{\text {Phe }}$ values, both indicative of marine protein consumption. Compared to other archaeological human isotopic compositions, the Rössberga humans plot closely to the $\mathrm{C}_{3}$ plant-derived protein consumers, the Köpingsvik humans cluster near the high marine protein consumers, and the Visby humans are intermediate between the two, although perhaps more similar to the $\mathrm{C}_{3}$ plant-derived terrestrial protein consumers.

\section{Applying a mixing model}

\section{Considerations for the IsoSource mixing model}

IsoSource has been widely used for ecological modeling (Phillips et al. 2005; Phillips and Gregg 2003), as well as some archaeological research (Beavan-Athfield et al. 2008; Beavan-Athfield and Mays 2009; Fabre et al. 2011; Grupe 2014; Mays and Beavan 2012; Newsome et al. 2004) and is both simple to use and freely available. Here, our goal is to use a mixing model to provide a more informative resolution of faunal and human $\delta^{13} \mathrm{C}_{\mathrm{E}-\mathrm{AA}}$ values. The isotopic compositions of five essential amino acids (phenylalanine, valine, isoleucine/leucine, and lysine) from fauna and humans from Visby and Köpingsvik were used to characterize differences in relative contributions of different dietary resources. Rössberga is not included because the sample is limited with respect to the number of faunal remains available for analysis, and also exhibited very low dietary variability.
Using essential amino acid isotopic compositions will mitigate the uncertainty resulting from the possibility of direct routing of nonessential amino acids and unknown tissue-diet relationships, because the essential amino acids must be routed from diet to tissue and are not extensively altered by isotopic discrimination during metabolism (Howland et al. 2003; Jim et al. 2006). Further, because $\delta^{13} \mathrm{C}_{\mathrm{E}-\mathrm{AA}}$ values do not change appreciably, their isotopic compositions will approximate consumed plant $\delta^{13} \mathrm{C}_{\mathrm{E}-\mathrm{AA}}$ values, thereby compensating to some extent for the limitations imposed by preservation in the archaeological record. The IsoSource model does not allow compensation for differences in the relative concentrations of the essential amino acids in the sources to be made. All the samples analyzed herein, however, are collagen, which minimizes the impact of this limitation. Fish collagens do typically have slightly higher concentrations of serine and glycine than mammalian collagens (Szpak 2011), but neither of these amino acids are used in the model. The $\delta^{13} \mathrm{C}_{\mathrm{E}-\mathrm{AA}}$ values should therefore enable a relative assessment of dietary inputs from different classes of local resources, e.g., marine vs. freshwater vs. terrestrial, and, to a lesser extent, among animals within these categories with different feeding ecologies. The human isotopic compositions (either sample averages or individuals) describe the "mixture" to be evaluated and the faunal isotopic compositions are the "source." The model then creates a mixing polygon that circumscribes all possible mixtures that are consistent with the isotopic compositions of the sources. In addition to the human and faunal isotopic compositions, the 
software requires the user to enter increment and tolerance parameters. Increment refers to the number of points evaluated at, for example, $1 \%$ intervals, whereas tolerance limits how far in per mil a solution can be outside of the mixing polygon and still be viable. Hypothetically, if the tolerance is not changed, decreasing the increment will increase the number of points evaluated and thus increase the number of solutions returned. In contrast, if the increment is not changed, decreasing the tolerance increases
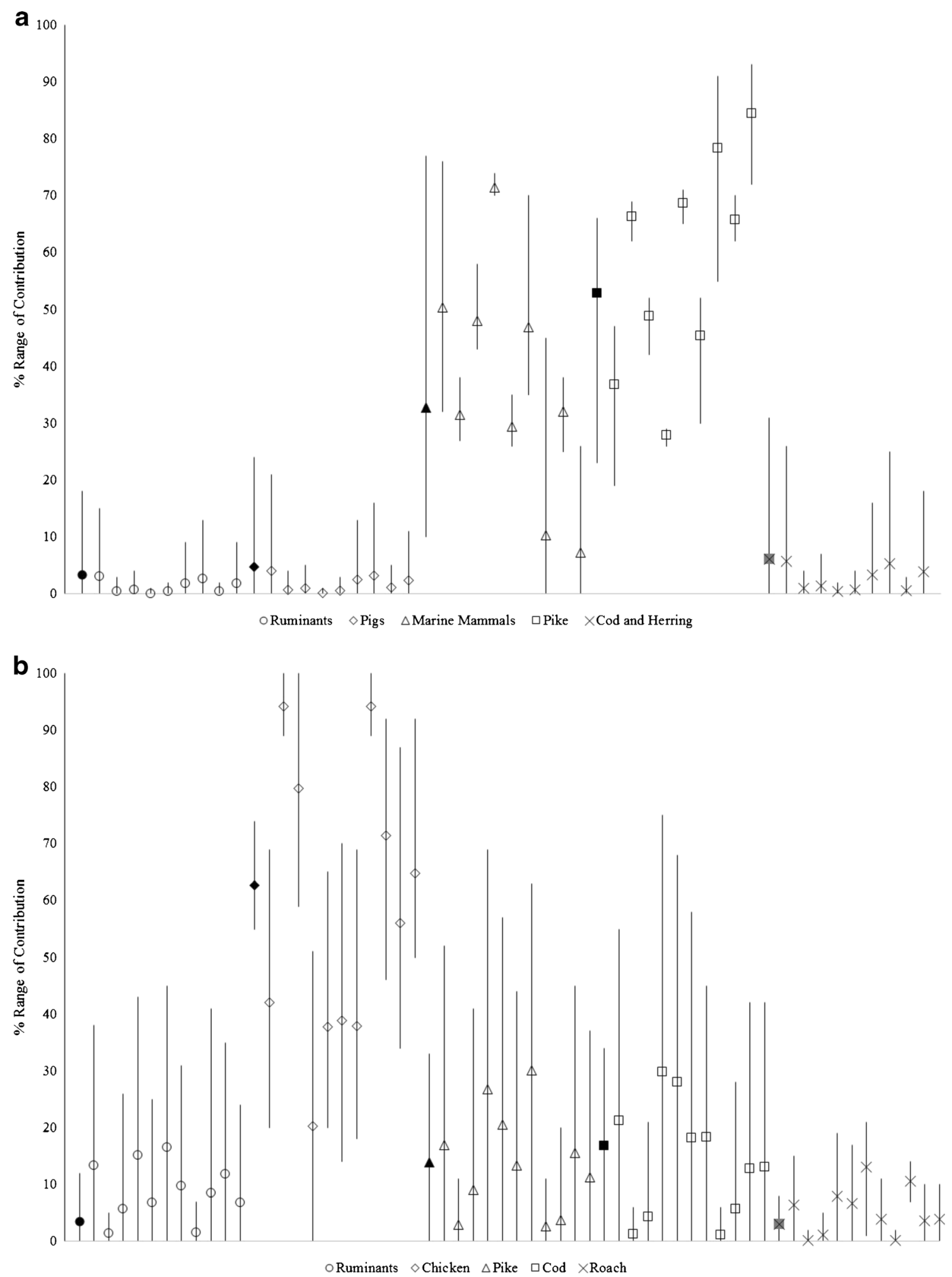

Fig. 8 Graphical representation of average and range of $\%$ contribution of different dietary protein sources generated using the $\delta^{13} \mathrm{C}_{\mathrm{E}-\mathrm{AA}}$ values for a Köpingsvik and b Visby. Filled squares: \% range of contribution for the sample; hollow circles: \% range of contribution for individuals 
Table 4 Comparison of mixing model dietary protein source contributions from bulk $\delta^{13} \mathrm{C}_{\mathrm{col}}$ and $\delta^{15} \mathrm{~N}_{\mathrm{col}}$ values vs. $\delta^{13} \mathrm{C}_{\mathrm{E}-\mathrm{AA}}$ values

\begin{tabular}{|c|c|c|c|c|c|c|c|}
\hline \multicolumn{4}{|l|}{ Bulk collagen model } & \multicolumn{4}{|c|}{ Compound-specific model } \\
\hline & Mean $(\%)$ & Minimum (\%) & Maximum (\%) & & Mean $(\%)$ & Minimum (\%) & Maximum (\%) \\
\hline \multicolumn{8}{|l|}{ Köpingsvik $^{\mathrm{a}}$} \\
\hline Terrestrial fauna & 2.2 & 0.0 & 6.0 & Ruminants & 3.4 & 0.0 & 18.0 \\
\hline Marine mammals & 90.1 & 79.0 & 100.0 & Pigs & 4.8 & 0.0 & 24.0 \\
\hline \multirow[t]{3}{*}{ Fish } & 7.7 & 0.0 & 18.0 & Marine mammals & 32.7 & 10.0 & 77.0 \\
\hline & & & & Pike & 52.8 & 23.0 & 66.0 \\
\hline & & & & Cod and herring & 6.2 & 0.0 & 31.0 \\
\hline \multicolumn{8}{|l|}{ Visby $^{\mathrm{b}}$} \\
\hline Ruminants & 39.8 & 29.0 & 50.0 & Ruminants & 3.5 & 0.0 & 12.0 \\
\hline Chickens & 58.0 & 47.0 & 70.0 & Chickens & 62.7 & 55.0 & 74.0 \\
\hline \multirow[t]{3}{*}{ Fish } & 2.2 & 0.0 & 6.0 & Pike & 13.9 & 0.0 & 33.0 \\
\hline & & & & Cod & 16.8 & 0.0 & 34.0 \\
\hline & & & & Roach & 3.1 & 0.0 & 8.0 \\
\hline
\end{tabular}

${ }^{\text {a }}$ For the bulk $\delta^{13} \mathrm{C}_{\mathrm{col}}$ and $\delta^{15} \mathrm{~N}_{\mathrm{col}}$ values, the tolerance is $1.5 \%$. For the $\delta^{13} \mathrm{C}_{\mathrm{E}-\mathrm{AA}}$ values, the tolerance is $2 \%$. These tolerance limits are the lowest values that produced viable solutions for the Köpingsvik mixture. Increment was set at $1 \%$ for both models

${ }^{\mathrm{b}}$ For the bulk $\delta^{13} \mathrm{Ccol}$ and $\delta^{15} \mathrm{Ncol}$ values, the tolerance is $0.5 \%$. For the $\delta^{13} \mathrm{CE}-\mathrm{AA}$ values, the tolerance is $0.2 \%$. These tolerance limits are the lowest values that produced viable solutions for the Visby mixture. Increment was set at $1 \%$ for both models

the accuracy of the solutions by excluding points far from the mixing polygon. In archaeological systems, which cannot be as well-understood as contemporary ecosystems, a low tolerance may be unrealistic and risks overinterpreting the data, whereas a too-wide tolerance risks rendering the model uninformative. In practice, IsoSource provides a set of viable solutions from different combinations of the source components that satisfy the isotopic mass balance model for each mixture at a particular tolerance level. Descriptive statistics (mean, minimum, and maximum) are generated by IsoSource and are used here to describe the distribution of the viable solutions.

For comparative purposes, bulk collagen $\delta^{13} \mathrm{C}$ and $\delta^{15} \mathrm{~N}$ values will also be assessed using IsoSource. To compensate for isotopic discrimination, tissue- and taxa-specific adjustments were made to the faunal collagen $\delta^{13} \mathrm{C}$ and $\delta^{15} \mathrm{~N}$ values to better approximate the isotopic composition of the edible portions (i.e., muscle) as follows: fish collagen $\delta^{13} \mathrm{C}$ values were adjusted downward by $0.8 \%$ (Dunn 2011) and mammalian collagen by $2.5 \%$ (Keegan and DeNiro 1988; unpublished data). No adjustments were made to faunal $\delta^{15} \mathrm{~N}_{\text {col }}$ values, since there is no consistent tissue-tissue isotopic offset between muscle and collagen, that is, $\delta^{15} \mathrm{~N}_{\text {col }} \approx \delta^{15} \mathrm{~N}_{\text {muscle. }}$. In order to better approximate the isotopic composition of consumed food, human $\delta^{13} \mathrm{C}_{\mathrm{col}}$ values were adjusted downward by $5 \%$ and $\delta^{15} \mathrm{~N}_{\mathrm{col}}$ values by $3 \%$ to compensate for tissue-diet discrimination and the trophic level effect, respectively (Ambrose 1993, 2000).

\section{Palaeodietary reconstruction using IsoSource}

Unless otherwise stated, all data in this section are reported as average [minimum-maximum]. Evaluated as a group, the human specimens from Köpingsvik showed generally low contributions of terrestrial (ruminant and pig) fauna and similarly low contributions of cod and herring to their total dietary protein (Fig. 8a, Table 4), but high contributions from pike $(\sim 53 \%)$ and marine mammals $(\sim 33 \%)$. At the individual level, however, there was some heterogeneity in the proportions of different protein sources contributing to total dietary protein. Terrestrial fauna and cod/herring inputs were somewhat higher for four individuals (KOP09, KOP30, KOP112, KOP120), but the average source contributions are still less than $10 \%$ for these resources. Highly variable amounts of marine mammal and pike were suggested, ranging from as low as $7 \%$ [0 to $26 \%$ ] for KOP120 and as high as $71 \%$ [70 to $74 \%$ ] for KOP70 for marine mammal consumption, and $28 \%$ [26 to $29 \%$ ] for KOP70 and $85 \%$ [72 to $93 \%$ ] for KOP120 for pike. Thus, although all individuals consumed some amount of marine protein, there is variability in the relative proportions of different sources of that marine protein that was not apparent using either bulk isotopic compositions or the compound-specific isotopic proxies. Using bulk carbon- and nitrogen-isotope compositions, IsoSource suggested a somewhat different dietary reconstruction for the humans from Köpingsvik ${ }^{3}$. Terrestrial contributions to diet were very low

\footnotetext{
${ }^{3}$ Because the number of source groups must equal the number of isotopic proxies used +1 , and because the number of proxies (i.e., $\delta^{15} \mathrm{~N}$ and $\delta^{13} \mathrm{C}$ values) is less than for the essential amino acid model $\left(\delta^{13} \mathrm{C}_{\mathrm{Phe}}, \delta^{13} \mathrm{C}_{\mathrm{Val}}\right.$, $\delta^{13} \mathrm{C}_{\mathrm{Lys}}, \delta^{13} \mathrm{C}_{\mathrm{Ile} / \mathrm{Leu}}$ ), source categories are grouped into terrestrial (ruminants + pigs), marine mammal, and fish (pike + cod + herring) for Köpingsvik, and ruminants, chickens and fish (pike + cod + roach) for Visby. These source combinations were chosen based on biological and ecological similarity among species, noting that while pike and roach are normally regarded as freshwater fish they co-exist within the brackish waters of the Baltic with cod, the latter normally being regarded as a marine species.
} 
( $\sim 2 \%)$, as were fish contributions $(\sim 8 \%)$, and much higher contributions of marine mammal protein was suggested ( $90 \%$; Table 3). This emphasis on higher trophic level marine organisms is consistent with current hypotheses regarding the impact of marine protein consumption on palaeodietary reconstruction, specifically that at higher levels of consumption, bulk collagen isotopic compositions overrepresent marine resource contribution to total dietary protein (Hedges 2004).

Human diet at Visby was dominated by terrestrial fauna, particularly chicken $(\sim 63 \%)$. There were low contributions generally from roach and ruminants ( $\sim 3 \%$ each). As with the humans from Köpingsvik, there is considerable diversity in relative source contributions (Fig. 8b, Table 3). Chickens generally contribute a considerable proportion of dietary protein for all individuals, ranging from $20 \%$ [0 to $51 \%$ ] for STH18 and $94 \%$ [89 to $100 \%$ ] for STM08 and STH31. Chickens consumed an omnivorous diet, including grains, grasses, small insects, and invertebrates (i.e., $\mathrm{C}_{3}$ plants and $\mathrm{C}_{3}$ plantconsuming fauna, albeit from a low trophic level). Because the model uses essential amino acid isotopic compositions, rather than indicating human consumption of a large amount of chicken, these data instead suggest common dietary resources between chickens and humans, i.e., terrestrial $\mathrm{C}_{3}$ plants and $\mathrm{C}_{3}$ plant-consuming fauna. Some individuals consumed very little aquatic protein (STH31: average 0.2 to $3 \%$ for three aquatic resources; STM03: 4 to $11 \%$, STM08:0.2 to $3 \%$ ) whereas others consume more, i.e., $30 \%$ average (STH18: 8 to $28 \%$, STM05: 4 to $30 \%$ ). It is likely that, as reflected in the $\delta^{13} \mathrm{C}_{\mathrm{AA}}$ proxies, most individuals were consuming a small amount of aquatic protein. In some cases, however, individuals may have been consuming considerably more when all three aquatic species are combined, e.g., STH18 (65\% from pike, cod and roach combined, and the remaining $35 \%$ from terrestrial sources). In contrast, STH31 acquired less than $5 \%$ of protein from marine sources and the remaining $95 \%$ from terrestrial resources. Thus, although the diet for Visby humans as a group can be described as including both terrestrial and marine resources, there are important differences in dietary behavior among individuals. Bulk carbon- and nitrogen-isotope compositions for the group again suggest a different dietary reconstruction. Terrestrial ruminant contributions were comparatively high ( $40 \%)$ and contributions from chickens were similar ( $58 \%)$, but marine resource contributions were considerably lower $(\sim 2 \%)$. This underrepresentation of marine resource contributions to total dietary protein supports the contention that, at low proportions of marine protein consumption $(<20 \%$ of total dietary protein), bulk collagen isotopic compositions mask the true extent of consumption (Hedges 2004).

Although IsoSource cannot provide a single solution for dietary source contributions for a group or individual, it does establish likely boundaries for different resources, as well as the relative frequencies of source contributions. Here, using
IsoSource enabled integration of a complex isotopic dataset and was especially informative about differences among individuals in dietary behavior. In a larger sample from an archaeological population, the kind of fine-grain description generated by the mixing model would be very valuable in understanding subtle changes in resource availability and in resource exploitation. Mixing model predictions based on the bulk $\delta^{15} \mathrm{~N}_{\mathrm{col}}$ and $\delta^{13} \mathrm{C}_{\mathrm{col}}$ values and based on the $\delta^{13} \mathrm{C}_{\mathrm{E}-\mathrm{AA}}$ values yielded substantially different palaeodietary reconstructions. These differences are likely due to variability in the extent of routing of non-essential amino acids, which may be dependent on the type of protein consumed (Corr et al. 2005). This limitation is to some extent addressed by using essential amino acid isotopic compositions. Further, the sample level essential amino acid model produces an arguably more realistic dietary reconstruction than using bulk isotopic data. For example, from $\delta^{13} \mathrm{C}_{\mathrm{col}}$ and $\delta^{15} \mathrm{~N}_{\mathrm{col}}$ values, the model indicates that humans from Köpingsvik relied heavily on marine mammals with minimal contributions from other aquatic and terrestrial resources. In contrast, using $\delta^{13} \mathrm{C}_{\mathrm{E}-\mathrm{AA}}$ values, the Köpingsvik diet incorporated a broad spectrum of marine resources, as well as moderately larger, although still small, contribution from terrestrial resources.

\section{Conclusion}

In this study, an array of $\delta^{13} \mathrm{C}_{\mathrm{AA}}$ isotopic proxies and relationships were used to enhance palaeodietary reconstruction at Rössberga, Köpingsvik, and Visby. All three proxies distinguished among humans from the three sites consistently and informatively, and were able to both confirm and further refine the broad interpretations made using bulk isotopic data. There is sometimes overlap in the $\delta^{13} \mathrm{C}_{\mathrm{E}-\mathrm{AA}}$ values for $\mathrm{C}_{3}$ and freshwater consumers in the same environment (e.g., continental Europe), or for marine and freshwater consumers, which could cause uncertainty using this relationship to distinguish among these consumer groups (Honch et al. 2012). Similarly, the $\delta^{13} \mathrm{C}_{\mathrm{Val}}$ values for $\mathrm{C}_{4}$ and marine consumers can also overlap, although they are typically distinguished by their $\delta^{13} \mathrm{C}_{\text {Phe }}$ values. Further research, ideally under controlled feeding conditions, would add rigor to the application of these proxies to more complex, and less well-constrained, dietary scenarios. Using IsoSource as a tool to integrate the complex amino acid isotopic dataset also proved highly informative at both sample and individual levels. The mixing model palaeodietary reconstruction was in general agreement with the inferences made based on the amino acid isotopic proxies, but, in addition, IsoSource revealed considerable diversity in relative protein source contributions among individuals at both Köpingsvik and Visby. Moreover, when the mixing model was used to assess diet from bulk carbon- and nitrogen-isotope compositions, a different dietary reconstruction was determined for 
both groups. This comparison of the mixing model outcomes for bulk collagen isotopic compositions vs. essential amino acid isotopic compositions demonstrated one of the fundamental limitations associated with bulk collagen isotopic analysis - the potential for underestimation and overestimation of marine protein consumption for both marine-dominant and mixed marine-terrestrial diets. Compound-specific amino acid isotopic analysis shows considerable promise as a means of refining palaeodietary reconstruction and, in conjunction with mixing models, may ameliorate the impact of several of the limitations associated with palaeodietary reconstruction using bulk carbon- and nitrogen-isotope compositions.

Acknowledgments This work was conducted as part of Responsive Mode grants from the UK Natural Environment Research Council (NERC: NE/D004535/1 and NE/010510/1). The NERC are also thanked for mass spectrometry facilities.

Open Access This article is distributed under the terms of the Creative Commons Attribution 4.0 International License (http:// creativecommons.org/licenses/by/4.0/), which permits unrestricted use, distribution, and reproduction in any medium, provided you give appropriate credit to the original author(s) and the source, provide a link to the Creative Commons license, and indicate if changes were made.

\section{References}

Ahlström, T. (2001) Det döda kollektivet: Skelettmaterialet från Rössbergagånggriften. In: Persson, P., Sjögren, K. -G. (eds.), Falbygdens gånggrifter: Del 1. Undersökningar 1985-1998 (Vol. 34, pp. 301-361), Göteborg.

Ambrose SK (1993) Isotopic analysis of palaeodiets: methodological and interpretive considerations. In: Sandford MK (ed) Investigations of ancient human tissue: chemical analyses in anthropology. Gordon and Breach Science Publishers, Langhorne, Pennsylvania, pp 59130

Ambrose SK (2000) Controlled diet and climate experiments on nitrogen isotope ratios of rats. In: Ambrose SH, Katzenberg MA (eds) Biogeochemical approaches to paleodietary analysis. Kluwer Academic/Plenum Publishers, New York

Ambrose SK, Norr L (1993) Experimental evidence for the relationship of the carbon isotope ratios of whole diet and dietary protein to those of bone collagen and carbonate. In: Lambert JB, Grupe G (eds) Prehistory human bone: archaeology at the molecular level. Springer-Verlag, London, pp 1-38

Beavan-Athfield N, Green RC, Craig J, McFadgen B, Bickler S (2008) Influence of marine sources on ${ }^{14} \mathrm{C}$ ages: isotopic data from Watom Island, Papua New Guinea inhumations and pig teeth in light of new dietary standards. J Roy Soc New Zeal 38:1-23

Beavan-Athfield, N., Mays, S. (2009) Amino-acid and stable isotope analysis: investigation of bone protein survival and dietary implications. In: Scull, C. (ed.), Early Mediaeval (Late 5th-Early 8th Centuries AD) Cemeteries at Boss Hall and Buttermarket, Ipswich, Suffolk. Society for Mediaeval Archaeology Monograph 27, Society for Mediaeval Archaeology, London, pp. $222-226$

Bogaard A, Heaton THE, Poulton P, Merbech I (2007) The impact of manuring on nitrogen isotope ratios in cereals: archaeological implications for reconstruction of diet and crop management practices. J Arch Sci 34(3):335-343
Brown TA, Nelson DE, Southon JR (1988) Improved collagen extraction by modified Longin method. Radiocarbon 30:171-177

Choy K, Smith CI, Fuller BT, Richards MP (2010) Investigation of amino acid $\delta{ }^{13} \mathrm{C}$ signatures in bone collagen to reconstruct human palaeodiets using liquid chromatography-isotope ratio mass spectrometry. Geochim et Cosmochim Acta 74:6093-6111

Corr LT, Sealy JC, Horton MC, Evershed RP (2005) A novel marine indicator utilising compound-specific bone collagen amino acid $\delta^{13} \mathrm{C}$ values of ancient humans. J Archaeol Sci 32:321-330

Corr LT, Richards MP, Jim S, Ambrose SH, Mackie A, Beattie O, Evershed RPE (2008) Probing dietary change of the Kwäday Dän Ts' ìnchị individual, an ancient glacier body from British Columbia: I Complementary use of marine lipid biomarker and carbon isotope signatures as novel indicators of a marine diet. J Archaeol Sci 35: 2102-2110

DeNiro MJ, Epstein S (1978) Influence of diet on the distribution of carbon isotopes in animals. Geochim et Cosmochim Acta 42: 495-506

DeNiro MJ, Epstein S (1981) Influence of diet on the distribution of nitrogen isotopes in animals. Geochim et Cosmochim Acta 42: 495-506

Dunn PJH (2011) Marine resource exploitation and consumption amongst ancient human populations: unravelling the carbon isotope signal of bone collagen. Unpublished $\mathrm{PhD}$ dissertation, University of Bristol

Dunn PJH, Honch NV, Evershed RP (2011) Comparison of liquid chromatography-isotope ratio mass spectrometry (LC/IRMS) and gas chromatography-combustion-isotope ratio mass spectrometry $(\mathrm{GC} / \mathrm{C} / \mathrm{IRMS})$ for the determination of collagen amino acid $\delta^{13} \mathrm{C}$ values for palaeodietary and palaeoecological reconstruction. Rapid Commun Mass Spec 25:2995-3011

Eriksson G, Linderholm A, Fornander E, Kanstrup M, Schoultz P, Olofsson H (2008) Same island, different diet: Cultural evolution of food practice on Öland, Sweden, from the Mesolithic to the Roman Period. J Anth Arch 27:520-543

Fabre, V., Condemi, S., Degioanni, A., Herrscher, E. (2011) Neanderthals versus modern humans: evidence for resource competition from isotopic modelling. International Journal of Evolutionary Biology, vol. 2011, Article ID 689315, 16 pages

Fogel M, Tuross N (2003) Extending the limits of paleodietary studies of humans with compound specific carbon isotope analysis of amino acids. J Archaeol Sci 30:535-545

Fraser RA, Bogaard A, Heaton T, Charles M, Jones G, Christensen BT, Halstead P, Merbach I, Poulton PR, Sparkes D, Styring AK (2011) Manuring and stable nitrogen isotope ratios in cereals and pulses: towards a new archaeobotanical approach to the inference of land use and dietary practices. J Archaeol Sci 38:2790-2804

Froehle AW, Kellner CM, Schoeninger MJ (2010) FOCUS: effect of diet and protein source on carbon stable isotope ratios in collagen: follow up to Warinner and Tuross (2009). J Archaeol Sci 37:2662-2670

Fuller, B.T., Fuller, J.T., Sage, N.E., Harris, D.A., O'Connell, T.C., Hedges, R.E.M. (2004) Nitrogen balance and $\delta^{15} \mathrm{~N}$ : why you're not what you eat during pregnancy. Rapid Commun Mass Spec. 18: 2889-2896

Fuller, B.T., Fuller, J.T., Sage, N.E., Harris, D.A., O’Connell, T.C., Hedges, R.E.M. (2005) Nitrogen balance and $\delta^{15} \mathrm{~N}$ : why you're not what you eat during nutritional stress

Fuller BT, Fuller JT, Sage NE, Harris DA, O'Connell TC, Hedges REM (2004b) Nitrogen balance and $\delta^{15} \mathrm{~N}$ : why you're not what you eat during pregnancy. Rapid Commun Mass Spec 18:2889-2896

Gröcke DR, Bocherens H, Mariotti A (1997) Annual rainfall and nitrogen-isotope correlation in macropod collagen: application as a palaeoprecipitation indicator. Earth and Planetary Sci Let 153:279-285

Grupe G (2014) Application of isotopic mixing models for palaeodietary and paleoecological studies. Anthropol Anz 71:21-39 
Hare P, Fogel M, Stafford T, Mitchell A, Hoering T (1991) The isotopic composition of carbon and nitrogen in individual amino acids isolated from modern and fossil proteins. J Arch Sci 18:211-292

Heaton THE, Vogel JC, von al Chevallerie G, Collet G (1986) Climatic influence on the isotopic composition of bone collagen. Nature 322: $822-823$

Hedges REM (2004) Isotopes and red herrings: comments on Milner et al. and Lidén et al. Antiquity 78:34-37

Hedges REM, Reynard LM (2007) Nitrogen isotopes and the trophic level of humans in archaeology. J Arch Sci 34:1240-1251

Hobson KA, Alisauskas RT, Clark RG (1993) Stable-nitrogen isotope enrichment in avian tissues due to fasting and nutritional stress: implications for isotopic analysis of diet. Condor 95:388-394

Honch NV, McCullagh JSO, Hedges REM (2012) Variation of bone collagen amino acid $\delta^{13} \mathrm{C}$ values in archaeological humans and fauna with difference dietary regimes: developing frameworks of dietary discrimination. Am J Phys Anthropol 148:495-511

Howland MR, Corr LT, Young SMM, Jones V, Jim S, van der Merwe NJ, Mitchell AD, Evershed RP (2003) Expression of the dietary isotope signal in the compound-specific $\delta^{13} \mathrm{C}$ values of pig bone lipids and amino acids. Intl $\mathrm{J}$ Osteoarchaeol 13:54-65

Jim S, Jones V, Ambrose SH, Evershed RP (2006) Quantifying dietary macronutrient sources of carbon for bone collagen biosynthesis using natural abundance stable isotope analysis. Br J Nutr 95: $1055-1062$

Jones V (2002) Investigating the routing and synthesis of amino acids between diet and bone collagen via feeding experiments and application to palaeodietary reconstruction. Unpublished $\mathrm{PhD}$ dissertation, University of Bristol

Keegan WF, DeNiro MJ (1988) Stable carbon-and nitrogen-isotope ratios of bone collagen used to study coral-reef and terrestrial components of prehistoric Bahamian diet. Am Antiquity 53:320-336

Kellner CM, Schoeninger MJ (2007) A simple carbon isotope model for reconstructing prehistoric human diet. Am J Phys Anthropol 133: $1112-1127$

Koch PL, Phillips DL (2002) Incorporating concentration dependence in stable isotope mixing models: a reply to Robbins, Hilderbrand and Farley (2002). Oecologia 133:14-18

Lidén K (1995) Megaliths, agriculture, and social complexity: a diet study of two Swedish megalith populations. J Anthropol Archaeol 14: 404-417

Linderholm, A. (2008) Migration in Prehistory: DNA and stable isotope analyses of Swedish skeletal material. Archaeological Research Laboratory, Stockholm University, Stockholm

Linderholm, A., Fornander, E., Eriksson, G., Mörth, C.-M., Lidén, K. (2014) Increasing mobility at the Neolithic/ Bronze Age transition - sulphur isotope evidence from Öland, Sweden. Internet Archaeology 37 (Human Exploitation of Aquatic Landscapes, special issue, eds. Ricardo Fernandes and John Meadows)

Lindkvist, J. (2008) Kyrkotillhörighet och diet i det medeltida Visby: Diet och social status utifrån isotopanalyser av gravlagda från tre Visbykyrkor

Mays S, Beavan N (2012) An investigation of diet in early Anglo-Saxon England using carbon and nitrogen stable isotope analysis of human bone collagen. J Arch Sci 39:867-874

McCullagh JSO, Juchelka D, Hedges REM (2006) Analysis of amino acid $\delta^{13} \mathrm{C}$ abundance from human and faunal bone collagen using liquid chromatography/isotope ratio mass spectrometry. Rap Comm Mass Spec 20:2761-2768

McCullagh JSO (2010) Mixed-mode chromatography/isotope ratio mass spectrometry. Rap Comm Mass Spec 24:483-494

Mekota A, Grupe G, Ufer S, Cuntz U (2006) Serial analysis of stable nitrogen and carbon isotopes in hair: monitoring starvation and recovery phases of patients suffering from anorexia nervosa. Rapid Commun Mass Spect 20:1604-1610

Naito Y, Honch NV, Chikaraishi Y, Ohkouchi N, Yoneda M (2010) Quantitative evaluation of marine protein contribution in ancient diets based on nitrogen isotope ratios of individual amino acids in bone collagen: An investigation at the Kitakogane Jomon site. Am J Phys Anthropol 143(1):31-40

Newsome SD, Phillips DL, Culleton BJ, Guilderson TP, Koch PL (2004) Dietary reconstruction of an early to middle Holocene human population from the central California coast: insights from advanced stable isotope mixing models. J Arch Sci 31:1101-1115

Olsen KC, White CD, Longstaffe FJ, Heyking K, McGlynn G, Grupe G, Rühli FJ (2014) Intraskeletal isotopic compositions $\left(\delta^{13} \mathrm{C}, \delta^{15} \mathrm{~N}\right)$ of bone collagen: nonpathological and pathological variation. Am J Phys Anthropol 153:598-604

Parkington J (1991) Approaches to dietary reconstruction in the Western Cape: Are you what you have eaten? J Arch Sci 18:331-342

Petzke KJ, Feist T, Fleig WE, Metges CC (2006) Nitrogen isotopic composition in hair protein is different in liver cirrhotic patients. Rapid Commun Mass Spec 20:2973-2978

Phillips DL (2012) Converting isotope values to diet composition: the use of mixing models. J Mammal 93:342-352

Phillips DL, Gregg JW (2003) Source partitioning using stable isotopes: coping with too many sources. Oecologia 136:261-269

Phillips DL, Newsome SD, Gregg JW (2005) Combining sources in stable isotope mixing models: alternative methods. Oecologia 144: $520-527$

Schwarcz HP, Dupras TL, Fairgrieve SI (1999) ${ }^{15} \mathrm{~N}$ enrichment in the Sahara: in search of a global relationship. J Archaeol Sci 26: 629-636

Smith CI, Fuller BT, Choy K, Richards MP (2009) A three-phase liquid chromatographic method for $\delta^{13} \mathrm{C}$ analysis of amino acids from biological protein hydrolysates using liquid chromatographyisotope ratio mass spectrometry. Anal Biochem 390:165-172

Szpak P (2011) Fish bone chemistry ultrastructure: implications for taphonomy and stable isotope analysis. J Archaeol Sci 38(12): 3358-3372

Styring AK, Sealy JC, Evershed RP (2010) Resolving the bulk $\delta^{15} \mathrm{~N}$ values of ancient humans and animal bone collagen via compound-specific nitrogen isotope analysis of constituent amino acids. Geohim Cosmochim Acta 74:241-251

Styring AK, Fraser RA, Arbogast R, Halstead P, Isaakidou V, Pearson JA, Schäfer M, Triantaphyllou S, Valamoti SM, Wallace M, Bogaard A, Evershed RPE (2015) Refining human palaeodietary reconstruction using amino acid $\delta^{15} \mathrm{~N}$ values of plants, animals and humans. J Archaeol Sci 53:504-515

White CD, Armelagos GJ (1997) Osteopenia and stable isotope ratios in bone collagen of Nubian female mummies. Am J Phys Anthropol 103:185-199

Williams LJ, White CD, Longstaffe FJ (2011) Improving stable isotopic interpretations made from human hair through reduction of growth cycle error. Am J Phys Anthropol 145:125-136 